Author's accepted manuscript deposited in the Open Access repository (http://real.mtak.hu/) of the Hungarian Academy of Sciences (HAS) by the author under obligation imposed by the HAS, in accordance with the Copyright Transfer Agreement. Copyright 2015, All Rights Reserved by Springer Science+Business Media Dordrecht. Publishing on other websites is not covered by the exemption granted to the author by the Copyright Transfer Agreement. Scientific use only. Warning: There are discrepancies between this version and the final published one.

Published in Husserl Studies 31/2 (2015), pp. 95-121.

"The final publication is available at Springer via http://dx.doi.org/10.1007/s10743-014-9155-z"

\title{
Was hat Husserl in Wien außerhalb von Brentanos Philosophie gelernt? Über die Einflüsse auf den frühen Husserl jenseits von Brentano und Bolzano
}

\author{
Peter Andras Varga*
}

\begin{abstract}
Husserl has undoubtedly considered himself being influenced by Brentano, but his conflicts with the orthodox core of the School of Brentano raise the question whether his adherence to Brentano suffices to adequately grasp the context of his early philosophy. I investigate the biographical details of Husserl's studies in Vienna to uncover hitherto unknown ties between Husserl and Austrian philosophers outside the School of Brentano. Already during his secondary school studies in the Austro-Hungarian Monarchy Husserl was exposed to the philosophy textbooks of Gustav Adolf Lindner; and archival records of the University of Vienna show that he had extensively attended philosophy courses held by other philosophers, especially by Robert Zimmermann. Both Lindner and Zimmermann proposed Herbartian philosophies, and what makes Zimmermann especially interesting is that he had once been a close personal disciple of Bernard Bolzano. I use an unpublished lecture transcript, written by a fellow student of Husserl, to investigate the controversial issue of Zimmermann's possible transmission of Bolzano's ideas. While a direct transmission seems improbable, my investigation uncovers a plethora of Herbartian influences, which are interesting on their own terms and have not only furnished Husserl with important parts of his descriptive psychological toolbox but have also helped him navigate the Brentano-School's debate on intentionality.
\end{abstract}

\section{Einleitung: Husserl und Brentano}

„Ohne Brentano hätte ich kein Wort Philosophie geschrieben“, soll Husserl 1932 einer Doktorandin gegenüber gesagt haben (Brück 1933, 3), die ihn zu seinem Verhältnis zu Brentano befragte. Auch wenn die Authentizität dieses Berichts nicht über jeden Zweifel erhaben ist, gibt er die schon damals weit verbreitete Ansicht anschaulich wieder, die auf die Beziehung von Husserl und seinem Lehrer, den er in Wien kennengelernt hat, das Schema eines Schüler-Meister-Verhältnisses anwandte. Dazu hat zweifellos am meisten Husserl selbst beigetragen. Denn in allen seinen Äußerungen über Brentano hat er ihn konsequent als seinen großen Lehrer bezeichnet, und in dem

* Husserl-Archiv der Universität zu Köln, Albertus-Magnus-Platz, D-50923 Köln. / Institut für Philosophie, Forschungszentrum für Geisteswissenschaften, Ungarische Akademie der Wissenschaften (MTA BTK FI), Orszaghaz u. 30, Budapest, Ungarn, E-Mail: Varga.Peter@btk.mta.hu 
Author's accepted manuscript deposited in the Open Access repository (http://real.mtak.hu/) of the Hungarian Academy of Sciences (HAS) by the author under obligation imposed by the HAS, in accordance with the Copyright Transfer Agreement. Copyright 2015, All Rights Reserved by Springer Science+Business Media Dordrecht. Publishing on other websites is not covered by the exemption granted to the author by the Copyright Transfer Agreement. Scientific use only. Warning: There are discrepancies between this version and the final published one.

Published in Husserl Studies 31/2 (2015), pp. 95-121.

"The final publication is available at Springer via http://dx.doi.org/10.1007/s10743-014-9155-z"

von Oskar Kraus 1919 herausgegebenen Nekrolog-Band findet man einen fast hagiografischen Erinnerungsbericht aus Husserls Feder. Sein bedingungsloses Bekenntnis zu Brentano sollte jedoch schon Kraus' Leser verwundern, da dieser in seiner Einleitung sogar Plagiatsvorwürfe gegen die anderen Brentano-Schüler erhoben hat: Ein Teil „,von Brentanoschem Lehrgute“ segelt „unter falscher Flagge“, und „Brentanos Anteil an den Publikationen seiner Schüler“ sollte überprüft werden. ${ }^{1}$ Allerdings hatte Husserl nicht nur zu dem orthodoxen Kern des Brentano-Schülerkreises ein gespanntes Verhältnis, sondern auch seine Beziehung zu Brentano scheint asymmetrisch gewesen zu sein. Brentano hat sogar zur Zeit seiner höchsten Sympathie für Husserl, nämlich in dem Empfehlungsbrief für Husserl an Carl Stumpf von 1886, zugestanden, „,noch keine Arbeit“" von Husserl gesehen zu haben (Brentano 1989, 84, vgl. 83); und als er Husserls Werke später näher kennengelernt hat, nahm seine ursprüngliche Sympathie drastisch ab. 1909 hat sich Brentano bei einer Berufungsangelegenheit sogar gegen Husserl ausgesprochen (s. 141). Es ist auch bemerkenswert, dass einige frühere Texte, die noch vor Kraus' meinungsbildendem Buch geschrieben wurden, Husserl nicht unbedingt mit Brentano assoziierten. ${ }^{2}$ All dies legt es nahe, das Schüler-Meister-Verhältnis zwischen Husserl und Brentano zu hinterfragen, um ein differenziertes Bild von Husserls Position zu gewinnen, die auch der Divergenzen Rechnung zu tragen versucht.

In meinem Beitrag möchte ich zunächst die historischen Aspekte offenlegen, die den jungen Husserl gerade mit Denkern außerhalb der Brentano-Schule verbunden haben. Diese Einflüsse, die nicht lediglich eine direkte Vermittlung des bolzanoschen Gedankenguts bedeuten, haben, wie ich nachzuweisen suche, Husserls spätere Auseinandersetzung mit der Brentano-Schule maßgeblich geprägt.

\section{Husserls Begegnungen mit der Philosophie in Wien}

\section{a) Vor der Universitätszeit}

1 Kraus 1919, 17. Ein Jahrzehnt später griff Kraus Husserl sogar namentlich an (s. Brentano 1930, xxi).

2 Dies berichtet Kraus selbst (Kraus 1919, 2 Anm.). In einer kürzlich aufgefundenen „Übersicht über die philosophischen Universitätsdocenten Deutschlands“ (Lotze 2003, 723), 1893 angefertigt von Hans Vaihinger, wurde Husserl definitiv nicht Brentano, sondern Stumpf als dessen „specieller Anhänger“ zugeordnet (Lotze 2003, 730). 
Author's accepted manuscript deposited in the Open Access repository (http://real.mtak.hu/) of the Hungarian Academy of Sciences (HAS) by the author under obligation imposed by the HAS, in accordance with the Copyright Transfer Agreement. Copyright 2015, All Rights Reserved by Springer Science+Business Media Dordrecht. Publishing on other websites is not covered by the exemption granted to the author by the Copyright Transfer Agreement. Scientific use only. Warning: There are discrepancies between this version and the final published one.

Published in Husserl Studies 31/2 (2015), pp. 95-121.

"The final publication is available at Springer via http://dx.doi.org/10.1007/s10743-014-9155-z"

Die Familie Husserl war mit der Kaiserstadt stark verbunden, die Edmund Husserl in seinen letzten Jahren immer wieder als ,alte Heimat“ bezeichnet hat. ${ }^{3}$ In den Wiener Adressbüchern ist Edmund Husserls Vater, (Abraham) Adolf Husserl, schon 1865 als „Handels-Agent“ verzeichnet, und seit 1879 hatte der älteste Bruder, Heinrich Husserl, der das 1860 in Proßnitz eröffnete väterliche Geschäft weitergeführt hat, auch eine „Offizielle Gesellschaft“ in Wien, die „Husserl-Brüder“, und später wurde eine Firma mit Sitz in Proßnitz und Wien gegründet. Im Laufe der Zeit sind noch mehrere Familienmitglieder nach Wien gezogen: Husserls Mutter, Julie Husserl (geb. Selinger), die 1917 in Wien starb, sowie Husserls Bruder Heinrich, der 1928 ebenso dort starb, und der jüngere Bruder Emil, der 1942 aus Wien deportiert wurde. Das Zentrum des Kaisertums Österreich war offenbar sowohl für die aus Proßnitz in Mähren stammende Familie Husserl als auch später für Husserls Frau, die ebenso aus Proßnitz stammende, aber in Klausenburg in Siebenbürgen geborene Malvine Steinschneider, ${ }^{4}$ eine naheliegende Wahl, da bis 1918 beide Städte zu der ÖsterreichischUngarischen Monarchie gehörten. Edmund Husserl selbst hat eine Mittelschule zunächst in Wien besucht, vermutlich deswegen, weil es in Proßnitz noch keine deutsche Mittelschule gab. Im Schuljahr 1868/69 ging Husserl in die erste Klasse des erst 1864 gegründeten Leopoldstädter Realund Obergymnasiums, ${ }^{5}$ das heute - nach einem anderen berühmten Schüler - Sigmund-FreudGymnasium heißt. ${ }^{6}$ Im nächsten Schuljahr wechselte Husserl an das deutsche Gymnasium in Olmütz, wo er bis zu seinem Abitur im Juni 1876 blieb. Im Unterschied zu seinen Brüdern hat er die inzwischen in Proßnitz eröffnete deutsche Mittelschule nie besucht.

Philosophische Bedeutsamkeit kommt dieser biografischen Episode dadurch zu, dass seit der Schulreform von 1849 ein Fach namens ,philosophische Propädeutik“ zum Lehrplan der österreichischen Gymnasien gehörte. Dieses Fach, das seit 1855 „formale Logik“ (im 7. Jahrgang)

3 BW IV, 206; BW IX, 81, 94, 101.

4 Über Husserls Abstammung siehe vor allem Kühndel 1969, über Malvine Husserls siehe Schuhmann 1988a, 105 ff.

5 Ms. X III 1. In dem Jahrbuch des Leopoldstädter Gymnasiums wird 1869 im Schülerverzeichnis der ersten Klasse ein „Husserl, Eduard“ genannt (Pokorny 1869, 82).

6 Dies bleibt nicht bloß eine biografische Kuriosität, indem die Freud-Forschung schon seit Langem darauf aufmerksam geworden ist, dass Freud nicht nur bei Brentano studiert hat, sondern auch herbartianischen Einflüssen ausgesetzt war (Hemecker 1991, 11 ff., 108 ff.), was eine ähnliche Untersuchung hinsichtlich Husserl fordert. In der Tat haben Freud und Husserl im Gymnasium anhand derselben Ausgabe eines herbartianischen Philosophielehrbuch studiert (siehe Anm. 8 unten). 
Author's accepted manuscript deposited in the Open Access repository (http://real.mtak.hu/) of the Hungarian Academy of Sciences (HAS) by the author under obligation imposed by the HAS, in accordance with the Copyright Transfer Agreement. Copyright 2015, All Rights Reserved by Springer Science+Business Media Dordrecht. Publishing on other websites is not covered by the exemption granted to the author by the Copyright Transfer Agreement. Scientific use only. Warning: There are discrepancies between this version and the final published one.

Published in Husserl Studies 31/2 (2015), pp. 95-121.

"The final publication is available at Springer via http://dx.doi.org/10.1007/s10743-014-9155-z"

und „empirische Psychologie“ (im 8. Jahrgang) umfasste, dürfte also Edmund Husserls erste nachweisbare Begegnung mit der Philosophie sein. Für den Unterricht der philosophischen Propädeutik in jenem Olmützer Gymnasium (wie übrigens auch im Leopoldstädter Gymnasium) wurden die in der Donaumonarchie weit verbreiteten Lehrbücher des herbartianischen Pädagogen und Philosophen Gustav Adolph Lindner ${ }^{7}$ verwendet. ${ }^{8}$ Der Lehrer für jenes Fach in den Schuljahren 1875-1876, und damit Husserls erster Lehrer in Philosophie überhaupt, war ein gewisser Anton Polzer. ${ }^{9}$ Über die in der philosophischen Propädeutik üblichen Lehrmethoden berichtet ein anderer Brentano-Schüler, der Pädagoge und Philosoph Alois Höfler, dass „,mir fast die Hälfte der Hörer meiner pädagogischen Vorlesungen und Übungen erzählte, bei ihnen sei ,der Lindner` Seite für Seite auswendig gelernt und vom Lehrer abgefragt worden“ (Höfler 1921, 122). Dies dürfte Husserl verständlicherweise wenig begeistert haben, wie seine relativ schlechten Schulnoten in jenem Fach (sowohl im 7-8. Jahrgang als auch im Abitur) zeigen. ${ }^{10}$ Trotzdem sollten wir nicht außer Acht lassen, dass die philosophische Propädeutik, besonders die logischen Grundbegriffe im Fach Formale Logik, einen prägenden Einfluss auf Husserl ausübte, den wir später, nämlich im Zusammenhang mit Husserls erneuerter Begegnung mit der österreichischen Schultradition der Logik, noch näher untersuchen werden.

7 Lindner (1828-1887) war mit den anderen Repräsentanten der herbartianisierenden Bolzano-Rezeption gut vernetzt (Grimm 2009, 23): Er studierte Philosophie bei dem Bolzanisten Franz Exner und besuchte das Priesterseminar zu Leitmeritz (Litoměřice), das bis zu dem Bolzanoprozess eine Hochburg des Bolzano-Kreises war, ferner hat er in Prag auch bei Robert Zimmermann studiert (Winter 1975, 27).

8 Siehe [Anonym] 1876, 55 (bzw. in dem früheren Jahrgang 44, aber ohne genauere bibliografische Angaben). Die dritte, erweiterte Auflage von Lindners Lehrbuch der formalen Logik erschien 1872, die vierte, neu durchgesehene Auflage erst 1877, so dass Husserl die dritte benutzen musste (wie übrigens auch Freud in Wien, siehe Hemecker 1991, 12 Anm. 14). In Husserls Bibliothek findet sich ein Exemplar der sechsten, revidierten Auflage (Signatur: BQ 258), das aber einen fremden Besitzvermerk trägt (,Hugo Liebing“). Es ist verständlich, dass Husserls eigenes Exemplar in seiner bewegten Studienzeit verloren gegangen ist; die Tatsache aber, dass er später ein neues Exemplar erworben hat, lässt auch auf die besondere Bedeutung Lindners für Husserl schließen.

9 Siehe [Anonym] 1876, 37 bzw. 39. Über Anton Polzer ist wenig bekannt. Laut sporadischen Erwähnungen in gedruckten Quellen des österreichischen Schulwesens wurde er am 19. Juni 1849 in Schönwald in Mähren (heute Šumvald in Tschechien) geboren, die Lehramtsqualifikation erwarb er im Schuljahr 1873/74 in Graz für klassische Sprachen mit Deutsch als Unterrichtssprache, als er schon Supplent (Hilfslehrer) in Olmütz war. Im Schuljahr nach Husserls Abitur wechselte jener zum zweiten staatlichen Gymnasium in Graz, wo er Deutsch und klassische Sprachen unterrichtete und 1890 befördert wurde. Alles deutet also darauf hin, dass für Polzer die Philosophie nur einen Nebenschauplatz seiner Lehrtätigkeit bildete.

10 Im 7. Jahr (1. und 2. Semester) erreichte Husserl noch ein „befriedigend“, aber sowohl im 8. Jahr als auch im Abitur hat er nur ein „genügend“ erreicht (Ms. X III 1), was in starkem Kontrast zu seinen guten Noten in naturwissenschaftlichen Fächern steht. 
Author's accepted manuscript deposited in the Open Access repository (http://real.mtak.hu/) of the Hungarian Academy of Sciences (HAS) by the author under obligation imposed by the HAS, in accordance with the Copyright Transfer Agreement. Copyright 2015, All Rights Reserved by Springer Science+Business Media Dordrecht. Publishing on other websites is not covered by the exemption granted to the author by the Copyright Transfer Agreement. Scientific use only. Warning: There are discrepancies between this version and the final published one.

Published in Husserl Studies 31/2 (2015), pp. 95-121.

"The final publication is available at Springer via http://dx.doi.org/10.1007/s10743-014-9155-z"

\section{b) Husserls Studium an der Universität Wien}

Nach alldem verwundert es nicht, dass Husserl 1881 nach seiner Studienzeit an deutschen Universitäten wieder nach Wien zurückkehrte, um sich auf seine Promotion in Mathematik vorzubereiten. Es ist jedoch erstaunlich, dass Husserls Studium an der Wiener Universität noch nicht untersucht wurde, obwohl das Archiv der Universität Wien detaillierte Quellen zu Husserls Studium aufbewahrt. ${ }^{11}$ Auf Grund der Immatrikulationsverzeichnisse der Universität Wien lassen sich nämlich Husserls Anmeldungen zu Lehrveranstaltungen genau rekonstruieren, die in den ersten zwei Semestern (aus einer anderen Quelle bekannt ${ }^{12}$ ) noch unproblematisch in unser herkömmliches Husserlbild passen: ${ }^{13}$

\section{Sommersemester 1881}

Königsberger, Leo: Differential- und Integralrechnung (II. Theil)

Königsberger, Leo: Zur Theorie der Differentialgleichungen

Königsberger, Leo <recte: Weyr, Emil>: Uebungen im mathematischen Seminar

Exner, Franz: Die Wellenbewegung in ihrer Erscheinung als Schall, Licht und

Wärme

Lang, Victor von: Theorie der Circularpolarisation

Brentano, Franz: Dialectische Uebungen (kritische Besprechung von Spinoza's

Ethik) ${ }^{14}$

11 Das Husserl-Archiv besitzt nur eine Kopie von Husserls Promotionsprotokoll und -akte aus dem Universitätsarchiv Wien (interne Signatur: X III 3).

12 Husserls Absolutorium (veröffentlicht in Gerlach - Rainer 1994, 166-167) wurde jetzt bestätigt und präzisiert. Die Anmeldungsliste von Husserls zweitem Studium in Wien ist dagegen bisher völlig unbekannt; ausschließlich die von Husserl angegebenen Lehrveranstaltungen (Schuhmann 1977, 13-16) wurden zitiert.

13 Die Liste habe ich auf Grund der von Husserl selbst ausgefüllten Immatrikulationsverzeichnisse (Ms. UA Wien Nationalen PHIL) rekonstruiert und vervollständigt auf Grund der genauen Titel- und Lehrkraftangaben der Vorlesungsverzeichnisse der Universität Wien.

14 Wie der Vordruck sagt, handelt es sich hier um das „Verzeichnis der Vorlesungen, welche der Studierende zu hören beabsichtigt", und in diesem Fall scheint die Annahme berechtigt zu sein, dass Husserl dies nicht verwirklicht hat, u. a. aus dem einfachen Grund, weil sich Brentanos Seminar mit Exners Vorlesung zeitlich überschnitt. Eine solche frühe Begegnung von Husserl und Brentano taucht in keiner späteren Erinnerung auf (ausgenommen bei Osbornes Interpretation: 1934, 16). Schon gemäß Husserls Nostrifikations-Lebenslauf von 1887 beginnt das „,me totum abdidi in studia philosopha [sic!] duce Francisco Brentano" erst 1884 (BW VIII, 222). Dieses Seminar fehlt auch im 1882 ausgestellten Absolutorium der Universität Wien (vgl. Anm. 12). 
Author's accepted manuscript deposited in the Open Access repository (http://real.mtak.hu/) of the Hungarian Academy of Sciences (HAS) by the author under obligation imposed by the HAS, in accordance with the Copyright Transfer Agreement. Copyright 2015, All Rights Reserved by Springer Science+Business Media Dordrecht. Publishing on other websites is not covered by the exemption granted to the author by the Copyright Transfer Agreement. Scientific use only. Warning: There are discrepancies between this version and the final published one.

Published in Husserl Studies 31/2 (2015), pp. 95-121.

"The final publication is available at Springer via http://dx.doi.org/10.1007/s10743-014-9155-z"

Wintersemester 1881/82

Königsberger, Leo: Theorie der elliptischen Functionen

Königsberger, Leo: Differential- und Integralrechnung (Fortsetzung)

Königsberger, Leo: Uebungen im mathematischen Oberseminar

Weyr, Emil: Neuere Geometrie (I. Theil)

Stefan, Joseph: Ueber Elektricität und Magnetismus

Stefan, Joseph: Uebungen im physikalischen Experimentieren

Im folgenden Sommersemester hat Husserl sich nicht immatrikuliert, sondern bekanntlich im Juli 1882 sein Promotionsgesuch in Mathematik eingereicht. Es ist jedoch wenig beachtet worden, dass der Verlauf von Husserls Promotionsverfahren eine wohl durchaus bemerkenswerte Lücke aufweist: Husserls Hauptrigorosum im Fach Mathematik fand am 29. November 1882 statt, sein Nebenrigorosum im Fach Philosophie aber erst am 15. Januar 1883. Zu Husserls Rigorosum waren die Rollen schon vertauscht: Er hat in der Philosophie ein deutlich besseres Ergebnis erzielt (und wurde mit dem Prädikat cum applausu approbiert) als in der Mathematik, wo er nur eine mäßige Leistung erbrachte. ${ }^{15}$ Über die Motive von Husserls Hinwendung zur Philosophie wurde viel spekuliert, ${ }^{16}$ aber sein zuerst verspätetes, dann aber mit einer hervorragenden Leistung abgeschlossenes philosophisches Nebenrigorosum dürfen wir vielleicht als das erste greifbare Zeichen seiner Umorientierung zur Philosophie verstehen. Jedenfalls war unter Husserls Prüfern ein Professor der Philosophie, der auch in Husserls späterem Werdegang wieder in Erscheinung tritt.

Husserls Interesse an Brentano dürfte durch Thomas Masaryk ausgelöst worden sein, der ihm laut indirekten Berichten (s. Schuhmann 1988a, 111, und Schuhmann 1988b, 137 Anm. 24) Brentano bereits in Leipzig empfohlen hat. Jedenfalls hat Husserl (gegen z.B. Schuhmann 1988b, 138 Anm. 26, und Rollinger 1999, 16) schon 1881 ein deutliches Zeichen seines Interesses mit seiner Anmeldung zu Brentanos Privatseminar für Fortgeschrittene gesetzt, die sich jedoch nicht realisieren ließ.

15 Im Hauptrigorosum erhielt Husserl zweimal ,ausgezeichnet“ (von Leo Königsberger und Emil Weyr), aber auch zweimal „genügend“ (von Victor von Lang und dem Historiker und Dekan Max Büdinger). Bei dem Nebenrigorosum bekam er dreimal ,,ausgezeichnet“ (von dem Philosophen Robert Zimmermann, dem Pädagogen Theodor Vogt und nochmals von Büdinger). Siehe Ms. UA Wien PHIL Rig Prot PN 268.

16 Husserl selbst schrieb den entscheidenden Impuls natürlich Brentano zu (d.h. er datiert ihn erst auf die Zeit nach seiner Promotion, s. Kraus 1919, 153), Husserls Frau hat dagegen über das „,schicksalhafte Resultat“ von Husserls Beschäftigung mit den Idealitäten der Mathematik während seiner Vorbereitung auf die Matura berichtet (Schuhmann 1988a, 110), in der neueren Literatur wiederum wurde der praktische Karriereaspekt betont (Rollinger 1999, 16). 
Author's accepted manuscript deposited in the Open Access repository (http://real.mtak.hu/) of the Hungarian Academy of Sciences (HAS) by the author under obligation imposed by the HAS, in accordance with the Copyright Transfer Agreement. Copyright 2015, All Rights Reserved by Springer Science+Business Media Dordrecht. Publishing on other websites is not covered by the exemption granted to the author by the Copyright Transfer Agreement. Scientific use only. Warning: There are discrepancies between this version and the final published one.

Published in Husserl Studies 31/2 (2015), pp. 95-121.

"The final publication is available at Springer via http://dx.doi.org/10.1007/s10743-014-9155-z"

Husserl hat sich, nach einer kurzen Zeit als inoffizieller Assistent von Karl Weierstraß und dem Militärdienst, erst im Wintersemester 1884/85 wieder immatrikuliert, aber schon seine erste Anmeldungsliste bietet einige Überraschungen, die später noch zunehmen sollten:

Wintersemester 1884/85

Zimmermann, Robert: Geschichte der Philosophie (III. Cursus: Neuere Zeit)

Brentano, Franz: Practische Philosophie

Brentano, Franz: Die elementare Logik und die in ihr nöthigen Reformen

Gomperz, Theodor: Geschichte der stoischen und epicureischen Philosophie,

dann Auswahl aus Lucrez

Vogt, Theodor: Encyklopädie der Philosophie

Zimmermann, Robert: Philosophisches Conversatorium

Brentano, Franz: In Gemeinschaft mit den Studierenden: Kritische Besprechung ausgewählter philosophischer Schriften ${ }^{17}$

Sommersemester 1885

Zimmermann, Robert: Logik

Zimmermann, Robert: Geschichte der Philosophie (IV. Cursus: Neueste Zeit)

Gomperz, Theodor: Aristoteles' Metaphysik (erlesene Abschnitte)

$<$ nicht angekündigt: $>{ }^{18}$ Brentano, Franz: Fortsetzung der Vorlesung und Seminar

über Hume (über die Prinzipien der Moral)

Wintersemester 1885/86

Brentano, Franz: Ausgewählte Fragen aus der Psychologie und Aesthetik

Brentano, Franz: In Gemeinschaft mit den Studierenden: Kritische Besprechung

von Laplace's philosophischem Versuch über die Wahrscheinlichkeiten ${ }^{19}$

17 Laut Husserls Erinnerungen wurde Humes Essay über den menschlichen Verstand behandelt (Kraus 1919, 155, vgl. Schuhmann 1977, 14).

18 Wegen seiner akademischen Herabstufung (s. Anm. 22 unten) hat Brentano ab SS 1884 in den Sommersemestern offiziell keine Lehrveranstaltung mehr angekündigt (die dementsprechend auch in dem Verzeichnis fehlen), hierzu bilden Husserls Erinnerungen (Kraus 1919, 153, 155) die einzige Quelle (vgl. Wieser 1950, 97; Antonelli 2001, 444).

19 Hier gibt Husserl Helmholtz’ Rede „Die Tatsachen der Wahrnehmung“ als Thema an (Kraus 1919, 155, interpretiert z.B. von Schuhmann 1977, 14, dagegen: Rollinger 1999, 17). Husserl dürfte Recht behalten, weil Brentano im nächsten Wintersemester wieder Laplace angekündigt hat (s. Antonelli 2001, 144), was für eine inoffizielle Themenänderung im WS 1885/86 spricht. 
Author's accepted manuscript deposited in the Open Access repository (http://real.mtak.hu/) of the Hungarian Academy of Sciences (HAS) by the author under obligation imposed by the HAS, in accordance with the Copyright Transfer Agreement. Copyright 2015, All Rights Reserved by Springer Science+Business Media Dordrecht. Publishing on other websites is not covered by the exemption granted to the author by the Copyright Transfer Agreement. Scientific use only. Warning: There are discrepancies between this version and the final published one.

Published in Husserl Studies 31/2 (2015), pp. 95-121.

"The final publication is available at Springer via http://dx.doi.org/10.1007/s10743-014-9155-z"

\author{
Zimmermann, Robert: Geschichte der Philosophie (I. Cursus: Alterthum) \\ Zimmermann, Robert: Philosophisches Conversatorium \\ Sommersemester 1886 \\ Freiherr von Berger, Alfred: Die Philosophie René Descartes' \\ $<$ nicht angekündigt: > Brentano, Franz: Fortsetzung der Vorlesung und Seminar \\ über Dubois-Reymond (Grenzen des Naturerkennens)
}

Das aus den archivalischen Quellen sich ergebende Bild von Husserls zweiter Studienphase in Wien weicht also erheblich von dem tradierten ab: Husserl hat nicht nur philosophische Lehrveranstaltungen auch bei anderen Lehrern als Brentano besucht (bei dem Pädagogen Theodor Vogt, dem Altphilologen Theodor Gomperz und dem Privatdozenten und Dichter Alfred Freiherr von Berger), sondern es gab noch einen anderen Philosophieprofessor, der für Husserl fast die gleiche Bedeutung hatte wie Brentano: der Hofrat Robert Zimmermann. ${ }^{20}$ Husserl hat bei ihm, der schon ein Prüfer im oben genannten philosophischen Nebenrigorosum war, nicht nur einen viersemestrigen Vorlesungszyklus über die Geschichte der Philosophie fast vollständig besucht, sondern auch an dessen Seminaren und Logik-Vorlesung teilgenommen. Husserls Begeisterung für Zimmermann wurde offensichtlich erst im vierten Semester getrübt, was darauf hindeuten kann, dass Husserls persönliche Beziehung zu Brentano, die 1886 z.B. in einen gemeinsamen Sommerurlaub am Wolfgangsee mündete, sich vielleicht erst Ende 1885 oder Anfang 1886 intensiviert hatte, ${ }^{21}$ da Brentano bei seinen Schülern bekanntlich keine fremden Lehrer duldete. ${ }^{22}$

20 Diese Tatsache war m.E. bisher unbekannt. Selbst gründlichste Darstellungen beschränken sich auf Zimmermanns allgemeine Rolle in Brentanos Umfeld (z.B. Rollinger 1999, 69 ff.), vielleicht ausgehend von einer vagen Vermutung Eduard Winters (BBGA E 1, 173). Rollinger $(1999,69)$ nimmt fälschlicherweise an, Husserl sei im Gymnasium Zimmermanns Logik-Lehrbuch begegnet.

21 Diese Feststellung passt gut zu der Tatsache, dass der viel zitierte Bericht über Husserl als ein ,neue[r] Stern auf dem philosophischen Himmel“ der Brentano-Schule nur vom 26.2.1886 stammt (Fabian 1986, 17).

22 Dies hat sogar Carl Stumpf in seinem wohlwollenden Nekrolog bemerkt (s. Kraus 1919, 146, vgl. Husserl, ibid., $160 \mathrm{ff}$.). Die archivalischen Quellen werfen also ein neues Licht auf eine bisher wenig beachtete Bemerkung in Brentanos Empfehlungsbrief, wo Brentano an Stumpf schrieb: „Daß er [Husserl] sich, durch keine Unterschiede der Stellung beirrt, nicht an Zimmermann, sondern aufs Engste an mich anschloß, darf ich wohl ohne zu große Unbescheidenheit als ein seinem Urteil günstiges Zeichen ansehen“ (Brentano 1989, 84). Zu Husserls Zeit war Zimmermann der einzige Philosophieprofessor in Wien, weil Brentano sein Katheder 1880 verloren hatte, als Folge seiner juristischen Manöver, um das in Österreich noch rechtskräftige Heiratsverbot für geweihte Priester zu umgehen. Brentanos Bemerkung dürfte also bis 1885/86 Wunschdenken geblieben sein und bestätigt, dass Husserls Anschluss an Zimmermann vorher eine ernstzunehmende Alternative bildete. 
Author's accepted manuscript deposited in the Open Access repository (http://real.mtak.hu/) of the Hungarian Academy of Sciences (HAS) by the author under obligation imposed by the HAS, in accordance with the Copyright Transfer Agreement. Copyright 2015, All Rights Reserved by Springer Science+Business Media Dordrecht. Publishing on other websites is not covered by the exemption granted to the author by the Copyright Transfer Agreement. Scientific use only. Warning: There are discrepancies between this version and the final published one.

Published in Husserl Studies 31/2 (2015), pp. 95-121.

"The final publication is available at Springer via http://dx.doi.org/10.1007/s10743-014-9155-z"

Die intensive Phase der persönlichen Beziehung Husserls zu Brentano dürfte also ausgesprochen kurz gewesen sein, da Husserl schon im Herbst 1886 nach Halle umzog. Es mag interessant sein, dass Husserl offenbar noch einen anderen maßgebenden Philosophielehrer in Wien hatte - vor allem, wenn wir hinzufügen, dass Robert Zimmermann ein enger persönlicher Schüler Bolzanos war und oft als Hauptvermittler von dessen Philosophie bezeichnet wurde.

\section{Zimmermann als Vermittler von Bolzano?}

\section{a) Zimmermanns Biografie}

Robert Zimmermann wurde 1824 in Prag geboren, hat 1846 in Wien promoviert und eine steile wissenschaftliche Karriere absolviert: Er habilitierte sich 1849 in Wien, aber statt an der Wiener Universität zu unterrichten, ging er im November 1849 als außerordentlicher Professor an die Universität Olmütz. 1852 wechselte er als ordentlicher Professor nach Prag und kehrte 1861 nach Wien zurück, wo er bis 1896, zwei Jahre vor seinem Tod amtierte. ${ }^{23}$ Zimmermann wurde dort zu einer einflussreichen Gestalt des Universitätslebens: Er war „wirkliches“ Mitglied der Akademie der Wissenschaften, Hofrat, später Regierungsrat, Rektor und - wie schon in Prag - Dekan (in starkem Kontrast zu Brentano, der seit 1880 eher ein akademischer Außenseiter war).

Obwohl der junge Husserl sich auf Grund Zimmermanns akademischer Stellung zu diesem hingezogen gefühlt haben mag, ist diese Beziehung doch vor allem wegen Zimmermanns vermutlicher Rolle als Hauptvermittler der bolzanoschen Philosophie unserer Aufmerksamkeit wert. Schon Zimmermanns Vater, Johann August Zimmermann (1793-1869), gehörte zum engsten Kreis Bolzanos, der seit dessen politischen Konflikten wie ein Geheimbund organisiert war und zielgerichtet die Verbreitung seiner Ansichten betrieb. ${ }^{24}$ Den jungen Robert Zimmermann hat

23 Über Zimmermanns Leben siehe besonders Alfred Wieser (1950, 74-83), der die sich auf Zimmermann beziehenden archivalischen Quellen der Universität erstmals ausgewertet hat.

24 Zimmermanns Vater nahm 1838 sogar an dem geheimen Treffen teil, wo die Geheimkodes für die Teilnehmer festgelegt wurden. Er schrieb Pamphlete und Rezensionen zur Verbreitung von Bolzanos Ansichten, obwohl dieser ihn für depressiv, faul und unzuverlässig hielt: Es sei ein „Wunder“, wenn etwas „,aus Zimmermanns Feder geflossen“ ist, und ein „zweites Wunder“, wenn darin Bolzanos Werke erwähnt wurden (BBGA III 3/3, 605). Allerdings hat Zimmermann sen. meist, wie z.B. 1843 in seinem Brockhaus-Lexikonartikel über Bolzano, gerade dessen für unsere Untersuchung relevanten Aspekte hervorgehoben: die „Unterschiede zwischen Vorstellung an sich 
Author's accepted manuscript deposited in the Open Access repository (http://real.mtak.hu/) of the Hungarian Academy of Sciences (HAS) by the author under obligation imposed by the HAS, in accordance with the Copyright Transfer Agreement. Copyright 2015, All Rights Reserved by Springer Science+Business Media Dordrecht. Publishing on other websites is not covered by the exemption granted to the author by the Copyright Transfer Agreement. Scientific use only. Warning: There are discrepancies between this version and the final published one.

Published in Husserl Studies 31/2 (2015), pp. 95-121.

"The final publication is available at Springer via http://dx.doi.org/10.1007/s10743-014-9155-z"

Bolzano früh kennengelernt und schnell zu seinem größten Hoffnungsträger aus ihm gemacht: „Zimmermanns Erstgeborener, der gegenwärtig schon in dem 2. Jahr der Philosophie ist, und sich einmal dem Lehrfache zu widmen gedenkt, entwickelt je mehr und mehr die seltensten Talente [...] auch zur Philosophie, hat sich schon jetzt mit einem großen Theile meiner Begriffe vertraut gemacht und brennt nur vor Begierde, Alles kennen zu lernen; [...] Ich setzte in der That vieles Vertrauen auf ihn $[\ldots]]^{\text {، } 25}$

Allerdings wurde Robert Zimmermanns biografisch nachvollziehbare Vermittlerrolle in der Forschung zunehmend infrage gestellt. ${ }^{26}$ Ein solcher Zweifel ist durchaus nicht unberechtigt, und schon Zimmermanns Zeitgenossen haben ihn als Herbart-Anhänger bezeichnet, wie 1889 auch Brentano (Brentano 1929, 130). Oskar Kraus hat dies in einem wenig bekannten Vortrag so formuliert: „auch der intime Schüler Bolzanos, [...] Robert Zimmermann, ging von Bolzano zu Herbart über“. ${ }^{27}$ Dies bestätigen auch seine öffentlichen Antrittsvorlesungen, die er kurz nacheinander in Olmütz, Prag und Wien gehalten hat. ${ }^{28}$

Die Hauptschwierigkeit bei der Klärung des Einflusses von Zimmermann auf Husserl besteht darin, dass er sich, obwohl er ein außerordentlich fruchtbarer Schriftsteller war, während seiner späteren Wiener Zeit, wo Husserls bei ihm studierte, zu den Kernthemen der Philosophie nur wenig geäußert

und Vorstellung als Seelenzustand [...]; jene als die Vorbedingung und den möglichen Stoff dieser.“(492)

25 BBGA III 3/2, 521, Robert Zimmermann sei ein „recht tüchtiges Werkzeug zur Verbreitung unserer Ansichten“ (547); vgl. auch 479-480; 3/3, 689.

26 Vgl. z.B. Morscher 1997 und Künne 2008, 316 ff.

27 Kraus 1935, 52. Kraus bezieht diesen Übergang gerade auf die für uns relevanteste philosophische Theorie: Bolzanos „Lehre von den sog. Vorstellungen, Sätzen und Wahrheiten an sich [...] scheint gerade jene seiner Freunde und Anhänger abgeschreckt zu haben, die sich der akademischen Lehrtätigkeit widmeten“" (ibid.).

28 Am Ende seiner Antrittsvorlesung in Olmütz bekannte sich Zimmermann noch ganz offen zu Bolzanos Ansichten: „Wissenschaftslehre ist der Inbegriff der Regeln, unser Wissen zur Deutlichkeit zu erhöhen, in einzelne Wissenschaften zu zerlegen, ihre Sätze als Gründe und Folgen aus und von einander zu begreifen und ihrem objektiven Zusammenhange nach zur Darstellung zu bringen. Als Lehre von den logischen Eigenschaften der Gedanken als Begriffe, Sätze und Satzverbindungen an sich betrachtet, ist sie zugleich die Fundamentalwissenschaft alles menschlichen, wie jedes Erkennens überhaupt [...]. Die Fragen daher: Gibt es überhaupt Wahrheiten? sind wir fähig, wenigstens einige derselben zu erkennen? und woran vermögen wir falsche Urtheile von wirklicher Erkenntnis zu unterscheiden? werden uns zunächst zu beschäftigen haben.“ (Zimmermann 1850, 16, vgl. 13) Zimmermanns Formulierungen, besonders seine Fragen, ähneln den Fragen, mit denen Bolzano den ersten Entwurf der Wissenschaftslehre in seinem Tagebuch 1812 konzipiert hat (s. unten Anm. 35). Aber in den späteren Antrittsvorlesungen treten solche Gedanken zunehmend in den Hintergrund, und Zimmermann geht zu einer eklektischen Philosophieauffassung über, die in der Pflege der regionalen philosophischen Disziplinen gipfelt (z.B. Zimmermann 1852, 4, 11; oder 1861, 3: Philosophie sei „nicht ein besonderes Wissen als vielmehr die Alles durchdringende forschende und ordnende Seele unseres Gesamtwissens“), was seinem späteren tatsächlichen Arbeitsfeld (Ästhetik) auch entspricht. 
Author's accepted manuscript deposited in the Open Access repository (http://real.mtak.hu/) of the Hungarian Academy of Sciences (HAS) by the author under obligation imposed by the HAS, in accordance with the Copyright Transfer Agreement. Copyright 2015, All Rights Reserved by Springer Science+Business Media Dordrecht. Publishing on other websites is not covered by the exemption granted to the author by the Copyright Transfer Agreement. Scientific use only. Warning: There are discrepancies between this version and the final published one.

Published in Husserl Studies 31/2 (2015), pp. 95-121.

"The final publication is available at Springer via http://dx.doi.org/10.1007/s10743-014-9155-z"

hat und auch kein Nachlass überliefert ist. Im Ausgang von einigen Hinweisen in Bolzanos Briefen wurde sogar die widerlegungsresistente Theorie entwickelt, Zimmermanns späteres Schweigen über Bolzano sei nur eine politisch bedingte Tarnung seines Bekenntnisses zu ihm. ${ }^{29}$

\section{b) Zimmermanns Vorlesungen über die Geschichte der neueren Philosophie}

Eine meines Erachtens bisher unbekannte Nachschrift von Zimmermanns Vorlesungen über die Geschichte der neueren Philosophie, ${ }^{30}$ angefertigt von dem späteren Wiener Kunsthistoriker Julius von Schlosser (1866-1938), stellt diesbezüglich eine bedeutsame Quelle dar, weil Schlosser gerade diejenigen Vorlesungen mitgeschrieben hat, die Husserl 1884-1885 bei Zimmermann gehört hat. Dies erweist sich aber als negativ, weil die Nachschrift jegliche direkte Vermittlung von Bolzano durch Zimmermann auszuschließen scheint. Eine Erwähnung Bolzanos lässt sich in der Nachschrift nicht finden.

Zimmermann teilt in jenen Vorlesungen die Geschichte der vorkantischen Philosophie in „continental-rationalistische“ und ,insular-realistische“ Strömungen ein, die sich in Kant „durchkreuzen und vereinigen.“31 Aus diesen Knotenpunkt gehen dann die ,idealistische“ und „realistische“ Strömung hervor. ${ }^{32}$ Zimmermanns Darstellung leidet offenbar an typischen Mängeln der Philosophiegeschichtsschreibung des 19. Jahrhunderts, wie etwa weltanschaulich belastete Etiketten, textferner Systematisierungszwang, Präsentialismus in der Darstellung etc. Sein Verdienst liegt jedoch in der Vermittlung eines Wissens über die Geschichte der Philosophie (was nicht zum Lehrplan der philosophischen Propädeutik gehörte). Vor allem seine relativ detaillierte Schilderung des Britischen Empirismus sollte hervorgehoben werden, weil sie auch Husserls langjährige Präferenz des Britischen Empirismus als Vorläufer der Phänomenologie zusätzlich Konturen

29 Bolzano hat den jungen Robert Zimmermann in der Tat gewarnt, seine Anhängerschaft zu verbergen (z.B. BBGA III 3/3, 707). Solche Bemerkungen dürfen aber nicht zu der generellen Annahme berechtigen, Zimmermanns Schweigen ohne Weiteres als ein unterdrücktes Bekenntnis zu Bolzano zu interpretieren (wie Eduard Winter, der Robert Zimmermann sogar noch 1979 einen ,geheime[n] Bolzanist[en]“ nannte, Winter 1993, 34). Zimmermanns späteres Schweigen über Bolzano ist viel leichter durch eine tatsächliche Meinungsänderung zu erklären (wenn Zimmermann überhaupt jemals Anhänger Bolzanos war).

30 Vorlesungsnachschrift von Julius v. Schlosser (im Teilnachlass von Julius v. Schlosser; Kunsthistorisches Museum Wien, Archiv, Sign. IV-64 / abgekürzt: Wien, KHM, AR IV-64).

31 Ibid., 1. Vorlesung, unpaginiert.

32 Ibid. 
Author's accepted manuscript deposited in the Open Access repository (http://real.mtak.hu/) of the Hungarian Academy of Sciences (HAS) by the author under obligation imposed by the HAS, in accordance with the Copyright Transfer Agreement. Copyright 2015, All Rights Reserved by Springer Science+Business Media Dordrecht. Publishing on other websites is not covered by the exemption granted to the author by the Copyright Transfer Agreement. Scientific use only. Warning: There are discrepancies between this version and the final published one.

Published in Husserl Studies 31/2 (2015), pp. 95-121.

"The final publication is available at Springer via http://dx.doi.org/10.1007/s10743-014-9155-z"

verleihen könnte. Zimmermann charakterisiert z.B. den Übergang von Locke zu Hume als Prozess einer schrittweisen Zurücknahme des Vorzugs primärer Eigenschaften:

Was ist aber der Körper dann, wenn wir seine Eigenschaften abstrahieren? [...] Wenn wir von einem Bündel Pfeile alle Pfeile wegnehmen, dann hört das Bündel auf. Die Realität hört auf, wenn alle Eigenschaften in Ideelles sich wandeln; was also übrig bleibt, ist das gerade Gegentheil, ein Unkörperliches, eine Welt von Ideen; statt wie Hobbes sagte: ,Nur der Körper ist.' lautet der Satz nun dahin: ,Nur die Vorstellung ist.' Aber was folgt dann? Wenn hinter der Vorstellung nichts ist, wenn hinter dem Vorhang nichts ist, dann ist der Vorhang eben keiner mehr, er ist selbst das, was ist. Hinter den Phänomenen ist nichts, sie haben uns kein Wesen aufzuschließen. ${ }^{33}$

Ein anderer hervorzuhebender Punkt ist die Würdigung der Mathematik als einzige Antwort auf die Herausforderung der humeschen Skepsis: „Die Mathematik ist unter allen möglichen Vorstellungsverbindungen über allen Zweifel [erhaben], weil über allem Zufall. Nur so weit [sic!] Mathematik in einer Wissenschaft ist, ist sie wissenschaftlich. ${ }^{\text {"34 }}$

Insofern ist Husserls Selbsteinschätzung widerlegt, er sei 1886, als er Wien verließ, ,ein krasser Anfänger in der Philosophie“ gewesen (Schuhmann 1988a, 113), widerlegt. Er dürfte vielmehr über eine nach zeitgenössischen Normen solide Grundausbildung in der Philosophiegeschichte verfügt haben, und eine Untersuchung von Husserls Kenntnissen der Geschichte der Philosophie muss auch Zimmermanns Vorlesungen in Betracht ziehen. Für Husserls unmittelbare Auseinandersetzung mit der Brentano-Schule wird aber seine Begegnung mit der österreichischen Schultradition der Logik, vermittelt sowohl durch Lindners als auch durch Zimmermanns Lehrbücher, von weitaus größerer Relevanz sein. Der Kontakt mit Zimmermann war für Husserl kein direkter Geheimweg zu Bolzano, diente aber als Bekräftigung einer logischen Tradition, die ihm schon durch Lindner vertraut war.

\section{Husserls Logik-Lehrbücher}

\section{a) Die philosophische Position von Lindners Logik-Lehrbuch}

33 Ibid., 2. Vorlesung.

34 Ibid., 3. Vorlesung. 
Author's accepted manuscript deposited in the Open Access repository (http://real.mtak.hu/) of the Hungarian Academy of Sciences (HAS) by the author under obligation imposed by the HAS, in accordance with the Copyright Transfer Agreement. Copyright 2015, All Rights Reserved by Springer Science+Business Media Dordrecht. Publishing on other websites is not covered by the exemption granted to the author by the Copyright Transfer Agreement. Scientific use only. Warning: There are discrepancies between this version and the final published one.

Published in Husserl Studies 31/2 (2015), pp. 95-121.

"The final publication is available at Springer via http://dx.doi.org/10.1007/s10743-014-9155-z"

Lindners Lehrbuch, das Husserl schon im Gymnasium begegnete, entspricht der herbartschen Vorlage sowohl im Aufbau als auch in der Bestimmung des „Begriff[s] des Begriffes.“ Diese zentrale Definition, die an der Spitze der Elementarlehre steht, lautete bei Herbart: Begriffe sind die Gedanken ,in Hinsicht dessen, was durch sie gedacht wird“ (Herbart 1813, 22). Herbarts Definition kehrt sinngemäß bei Lindner wie folgt wieder: „Den Begriff eines Gegenstandes angeben heißt [...] so viel, als die Frage beantworten, was dieser Gegenstand sei, oder was man sich unter demselben zu denken habe“" (Lindner 1872, 9). Die herbartianische Weise der Definition des Begriffs mag künstlich wirken, aber Lindner führt zwei Gründe an, die andere, sich leicht anbietende Definitionen als unzureichend erscheinen lassen. Begriffe können nicht einfach als „Zusammenfassung der wesentlichen Merkmale eines Dinges“ definiert werden (10), weil es auch nicht-zusammengesetzte Begriffe gibt. Begriffe können aber auch nicht als das aufgefasst werden, was bei verschiedenen Gegenständen das Gemeinsame ist, weil es auch Individualbegriffe (Einzelbegriffe) gibt, denen nur ein einziger Gegenstand entsprechen soll, wie z.B. der (monotheistische) Begriff Gott. Diese trivialen Definitionsversuche sind in der Tat auszuschließen, jedoch bedeutet dies nicht, dass die herbartianische Definition unproblematisch ist. Aus moderner Sicht lassen sich vor allem zwei problematische Aspekte dieser Definition hervorheben: Einerseits tendiert sie dazu, den Unterschied zwischen Begriffen und Vorstellungen zu verwischen, andererseits bleibt die Art und Weise der Gegenstandsbeziehung völlig im Dunklen.

In beiden Hinsichten sind sowohl die bolzanosche als auch die brentanosche Definition besser positioniert. Für Bolzano bilden die Wahrheiten an sich den Ausgangspunkt, die eindeutig als extramentale ideale Entitäten aufgefasst werden können. ${ }^{35}$ Die Vorstellungen werden dann als unselbstständige Teile dieser Wahrheiten definiert und die Begriffe als Vorstellungen, die nicht anschaulich sind und auch keine Anschauungen als Teile enthalten, wobei die anschaulichen Vorstellungen mit Hilfe des Inhalts und Umfangs rein logisch abgegrenzt werden können. Infolge

35 Dies wird besonders in Bolzanos erstem Entwurf der Wissenschaftslehre in einer Tagebuchnotiz um 1812 deutlich: „1 Capitel. Es gibt Wahrheiten. [...] 2 Capitel. Wir sind im Besitze der Erkenntnis gewisser Wahrheiten. [...] 5 Capitel. Es gibt unter den Wahrheiten einen objektiven Zusammenhang." (BBGA IIB 16/1, 34-35). In der Wissenschaftslehre selbst wird zuerst der Begriff des Satzes an sich eingeführt (BBGA I 11/1, 103) und nach der Begriffserklärung des Adjektivs ,wahr“ werden die Wahrheiten an sich als eine Art der Sätze an sich bestimmt (BBGA I 11/1, 137). 
Author's accepted manuscript deposited in the Open Access repository (http://real.mtak.hu/) of the Hungarian Academy of Sciences (HAS) by the author under obligation imposed by the HAS, in accordance with the Copyright Transfer Agreement. Copyright 2015, All Rights Reserved by Springer Science+Business Media Dordrecht. Publishing on other websites is not covered by the exemption granted to the author by the Copyright Transfer Agreement. Scientific use only. Warning: There are discrepancies between this version and the final published one.

Published in Husserl Studies 31/2 (2015), pp. 95-121.

"The final publication is available at Springer via http://dx.doi.org/10.1007/s10743-014-9155-z"

dieser klaren ontologischen Verortung von Vorstellungen und Begriffen gelingt es Bolzano, deutlich zwischen Begriffen, deren subjektiven Realisationen und Gegenstandsbeziehungen zu differenzieren. Ähnlich positiv erscheint die brentanosche Auffassung, die, systematisch dargestellt in den deskriptiven psychologischen Vorlesungen von Anton Marty, Begriffe konsequent als eine Art von Vorstellungen versteht, die mit Hilfe einer rein psychischen Abstraktionstheorie zu begrenzen sind; ${ }^{36}$ und die Gegenstandsbeziehung galt schon 1874 in Brentanos Durchbruchswerk als das positive Hauptmerkmal der psychischen Phänomenen.

Das Fehlen von solchen konsequenten Differenzierungen war bereits in Herbarts Lehrbuch spürbar, wo Begriffe und psychische „Thätigkeiten“ als zwei „Seiten“ der „Gedanken“ konzipiert wurden (Herbart 1813, 21). Auch Lindner kämpft offenbar vergeblich um die Begründung des Unterschieds zwischen Vorstellungen und Begriffen. Er sieht, dass Begriffe, im Gegensatz zu Vorstellungen, nicht mehrfach realisiert werden können und sich sowohl ontologisch als auch nach ihrer Zeitform von Vorstellungen unterscheiden; allerdings kann er die Begriffe bestenfalls mit einem ,abstracte[n] Muster" vergleichen (Lindner 1872, 11). Aus Lindners Bemühungen um die ontologische Verortung der Begriffe ergeben sich allerdings einige bemerkenswerte Auswirkungen, indem er versucht, die Funktion des Begriffs im Denken näher zu bestimmen:

Der Begriff ist also das geistige Gegenstück (Correlat) eines außerhalb des Denkens gesetzten, wirklichen oder möglichen, ja selbst unmöglichen Objectes - oder Dasjenige, was diesem Object in unserem Denken entspricht und wodurch wir es von jedem anderen Objecte geistig unterscheiden. $^{37}$

Dies kann zwar zur Klärung des ontologischen Status des Begriffs nichts beitragen, stellt aber eine wichtige Tendenz in Lindners Logik dar: die Tendenz zur Hypostasierung eines „logischen Objekts“. Denn Lindner scheut sich nicht, die logischen Objekte explizit als eine neue Klasse neben den Begriffen einzuführen: „Die Worte »Tugend«, »Glätte«, »Neptun« [...] bezeichnen nicht bloß Begriffe, sondern auch gewisse logische Objecte, gleichgiltig, ob ihnen in der realen Welt etwas entspricht oder nicht“ (Lindner 1872, 10). Die logischen Objekte, mit deren Hilfe er die Definition

36 Vgl. Marty 2011, 132. Marty versucht sogar, den synthetischen (nicht auf Abstraktion beruhenden) Momenten des Begriffsbildungsprozesses Rechnung zu tragen.

37 Lindner 1872, 9. 
Author's accepted manuscript deposited in the Open Access repository (http://real.mtak.hu/) of the Hungarian Academy of Sciences (HAS) by the author under obligation imposed by the HAS, in accordance with the Copyright Transfer Agreement. Copyright 2015, All Rights Reserved by Springer Science+Business Media Dordrecht. Publishing on other websites is not covered by the exemption granted to the author by the Copyright Transfer Agreement. Scientific use only. Warning: There are discrepancies between this version and the final published one.

Published in Husserl Studies 31/2 (2015), pp. 95-121.

"The final publication is available at Springer via http://dx.doi.org/10.1007/s10743-014-9155-z"

des Begriffs zu stützen versucht, haben also den eindeutigen Vorzug, dass sie unabhängig von dem ontologischen Status des eigentlichen Objekts der Vorstellung zur Verfügung stehen können: „es [ist] nicht unbedingt notwendig, daß die »logischen Objecte« wirklich (reale) Dinge sind; sie unterscheiden sich vielmehr von den »metaphysischen Objecten« dadurch, daß sie bloße Gedankendinge sind“ (Lindner 1872, 9). Dies hebt Lindner mit einem Zitat von Moritz Drobisch hervor, das auch unsere Aufmerksamkeit verdient: „Die Fabelwesen der Mythologie lassen sich so gut wie Naturkörper definieren, classificiren, vergleichen, sie haben ebenso gut wie diese eigenthümliche Merkmale und abgeleitete Eigenschaften u. s. f.“.${ }^{38}$ Die Fähigkeit unseres Denkens, sich auf nicht existierende Gegenstände zu beziehen, führte also Drobisch wie Lindner letztlich zu der Annahme eines ontologisch unabhängigen Reiches der logischen Objekte: „die Objecte der Logik sind an sich nichts mehr als Gedankendinge, denen zwar reale Objecte entsprechen können, aber nicht müssen“ (Drobisch 1863, 15).

Das plötzliche Auftreten der ontologisch unabhängigen logischen Objekte in einer herbartianischen empirischer Denkpsychologie ist umso bemerkenswerter, als Lindner (wie natürlich auch Drobisch) über eine klare Definition des Inhalts und Umfangs der Begriffe verfügt, die damals zum Gemeingut der traditionellen Logik gehörte. ${ }^{39}$ Das logische Objekt ist also keineswegs ein Ersatz für die fehlende Inhalt-Umfang-Unterscheidung, sondern kann als ein spezifisches Merkmal betrachtet werden, dem wir bei Husserl später wieder begegnen werden. Dieser muss mit Lindners Lehrbuch von seiner Schulausbildung her vertraut gewesen sein, aber auch Zimmermanns LogikLehrbuch, das sich in mehreren Ausgaben in Husserls Besitz befand, ist ein näherer Einblick wert.

\section{b) Das Logik-Lehrbuch Zimmermanns und Husserls explizite Auseinandersetzung mit ihm}

Zu seinem Verhältnis zu Herbart hat sich Zimmermann in den wenig beachteten Vorworten seiner Lehrbücher geäußert. Die erste Ausgabe von 1852, die Husserl anscheinend nicht besaß, entsprach noch weitgehend der Wissenschaftslehre Bolzanos. ${ }^{40}$ Die zweite Auflage von 1860, die in Husserls

38 Lindner 1872, 9, die zitierte Stelle findet sich bei Drobisch 1863, 15.

39 Siehe Lindner 1872, 15, 26.

40 Siehe die Auflistung der erheblichen Überlappungen bei Winter 1975. Sein zugespitztes Urteil, die erste Auflage sei deshalb ein „Plagiat“" gewesen (Winter 1975, 5, vgl. 22), ergibt sich aus einer illegitimen Rückprojektion 
Author's accepted manuscript deposited in the Open Access repository (http://real.mtak.hu/) of the Hungarian Academy of Sciences (HAS) by the author under obligation imposed by the HAS, in accordance with the Copyright Transfer Agreement. Copyright 2015, All Rights Reserved by Springer Science+Business Media Dordrecht. Publishing on other websites is not covered by the exemption granted to the author by the Copyright Transfer Agreement. Scientific use only. Warning: There are discrepancies between this version and the final published one.

Published in Husserl Studies 31/2 (2015), pp. 95-121.

"The final publication is available at Springer via http://dx.doi.org/10.1007/s10743-014-9155-z"

Besitz war und die er auch annotiert hat, hat Zimmermann aber so stark modifiziert, dass er selbst im Vorwort zur dritten Auflage die zweite schon als neues Buch bezeichnet hat. ${ }^{41}$ Es ist kaum zu übersehen, dass diese Veränderung vor allem in einer Annäherung an Herbart besteht. Den für uns wichtigsten Schritt dieses Übergangs erläutert Zimmermann in dem Vorwort zur zweiten Auflage wie folgt:

Es ist derselbe Unterschied, den der Verfasser bisher mit Bolzano's Wissenschaftslehre durch die Bezeichnungen ,Vorstellungen an sich` und ,subjective Vorstellung ${ }^{6}[\ldots]$ auszudrücken gesucht hat. Wenn er in dieser Auflage mit Herbart [...] die Vorstellung im logischen Sinne ,Begriff‘, die im psychischen ,Vorstellung' schlechtweg nennt [...], so tut er das, nicht weil er seine Ansichten geändert hat [...]. ${ }^{42}$

Zimmermanns tatsächliche Loyalität gegenüber Bolzano brauchen wir hier nicht zu bewerten. Sein Bekenntnis hebt aber das für Husserl relevanteste Moment seiner Annäherung an Herbart hervor: den Übergang von einem bolzanoschen Schema $\mathrm{zu}$ einer instabilen herbartianischen Unterscheidung.

Im Husserl-Nachlass findet sich ein Manuskript, in dem Husserl Zimmermanns Lehrbuch teilweise exzerpiert und sich mit ihm auseinandergesetzt hat. ${ }^{43}$ Hierbei interessierte sich Husserl gerade für Zimmermanns Definition des Begriffs: „Was ist ein Begriff?“, fragt dieser in dem von Husserl exzerpierten Teil und antwortet in ganz herbartianischer Weise: „Dasjenige, was eigentlich gedacht werden soll, wenn man eine gewisse psychische Vorstellung[,] z.B. diejenige hat, welche man mit dem Worte ,Baum‘ bezeichnet.“44 Zimmermann hatte aber ein spezielles Problem zu erörtern, nämlich die Möglichkeit eines inhaltlichen Unterschiedes zwischen dem Begriff und der ihn realisierenden subjektiven Vorstellungen: „Wer niemals Palmen gesehen hat: Für ihn gehört zum Begriff Baum ,Zweige haben“. Dieses Merkmal verschwindet, wenn er Palmen kennenlernt.“45 Oder in einem anderen Beispiel, das Husserl ebenso exzerpiert hat: „,Wer nie gelernt hat, beim Begriff des Dreiecks von dem Merkmal der Ebenheit abzusehen, <dem> lässt sich nicht begreiflich machen,

wissenschaftlicher Standards auf die intellektuellen Verhältnisse des frühen 19. Jahrhunderts der Donaumonarchie.

41 Zimmermann 1867, iii (die dritte Auflage war zwar auch in Husserls Besitz, weist aber keine Lesespuren auf).

42 Zimmermann 1860, vii.

43 Ms. K I 59/2a-2b. Die exzerpierten Stellen sind in Husserls Exemplar meist am Rand rot gekennzeichnet.

44 Zimmermann 1860, 17 (exzerpiert von Husserl: K I 59/2a).

45 Zimmermann 1860, 17, zitiert nach: K I 59 / 2a. 
dass es auch sphärische Dreiecke gibt. Winkelsumme ist $>2 R .{ }^{\text {، } 46}$

Zimmermanns Lösung bestand darin, den logischen Begriff als ein Ideal zu interpretieren „,nach welchem die psychische Vorstellung sich bequemen soll [...]. Alle Versuche, den Baum zu denken, zielen auf das Denken des logischen Begriffs vom Baume ab, indem sie durch Hinweglassen dieser, Hinzufügen jener Bestandtheile ihre psychische Vorstellung [...] dem logischen anzupassen suchen“ (Zimmermann 1860, 18). Das Verhältnis zwischen dem Begriff und der ihn realisierenden Vorstellung ist laut Zimmermann wie dasjenige zwischen einem „künstlerischen Ideal“" und dessen Nachbildungen, z.B. durch Statuen. Husserl hat diesen Lösungsversuch zuerst wörtlich mitgeschrieben, dann aber ausdrücklich abgelehnt:

Danach wird man doch sagen müssen, dass der logische Begriff eine zweite Vorstellung ist neben ihren Nachbildungen. Denn wie könnten wir sonst von einem Ideal reden? Wie soll $<\mathrm{t}>\mathrm{en}$ wir sagen, dass mehrere Vorstellungen dasselbe, denselben Begriff denken? Denkt derjenige, welcher zum Begriff des Dreiecks die Ebenheit rechnet, denselben Begriff wie derjenige, welcher es nicht tut? $?^{47}$

Husserl weist hier mit Recht darauf hin, dass, selbst wenn ein solcher variativer Approximationsprozess im Denken in der Tat vorkommt, er zum Verständnis der Gegenstandsbeziehung der Vorstellungen bzw. des Unterschieds zwischen Begriffen und Vorstellungen nichts beitragen würde. In den kommenden Jahren wird Husserl noch regelmäßig zu diesem Problem zurückkehren, nämlich auf Grund seiner neu erarbeiteten konzeptuellen Distinktionen. $^{48}$

\section{Die Auswirkungen der Einflüsse}

Zimmermanns und Lindners Bedeutung erweist sich vor dem Hintergrund der veröffentlichten Werke Husserls und seines Nachlasses als eher peripher ${ }^{49}$ (was auch erklären kann, warum dies

46 Zimmermann 1860, 18, zitiert nach: K I 59 / 2b.

47 Ms. K I 59 / 2b.

48 Hua XIX/1, 527; ohne Nennung von Namen: Hua XXII, 349 Anm., Ms. K I 61 / 5 a.

49 Lindners Name kommt anscheinend nur einmal, nämlich in Husserls Logik-Vorlesung vom WS 1910/11 vor (Hua XXX, 337, 531; der Name fehlt auch im Namensregister), wo Husserl seinen Studenten Lindners Lehrbuch empfahl. 
bisher nahezu übersehen wurde). Aber die oben angestellten historischen Überlegungen werfen nun ein neues Licht auf Husserls häufige, aber gleichwohl meist vernachlässigten Hinweise auf Logikautoren seiner Zeit, hinter denen sich oft die logische Tradition verbirgt, die er in seiner Wiener Schul- und Studienzeit begegnete.

\section{a) Erste Entfaltung von Husserls deskriptiv-psychologischen Interessen}

Spuren dieser Einflüsse können wir in einem Text vom Wintersemester 1893/94 finden, der zu der Reihe von Arbeiten gehört, die Husserl nach erfolgloser Arbeit an seinem Raumbuch verfasst hat, um für sich selbst die allgemeineren Fragen der deskriptiven Psychologie zu klären. Sein Ausgangspunkt ist dabei die Anwendung der Existenzialurteile auf die zwei Klassen von Vorstellungen, die er in anderen Texten dieser Periode schon klar voneinander abgegrenzt hat: ${ }^{50}$ auf Anschauungen (ich sage ,,vom gesehenen Schreibtisch ,Er existiert“") und Repräsentationen (,,[i]ch ,denke' an den Schreibtisch und urteile ,Er existiert'“, Hua XL, 31). Dieser Unterschied bereitet Husserl jetzt Schwierigkeiten, weil die entsprechenden Existenzialurteile zusammenzufallen scheinen:

Würde ich [bei den anschaulichen Existenzialurteilen] meinen[,] das Ding an sich, wie immer ich es fasse, sei es auch als ein Ding, das genauso, wie ich es sehe, unabhängig von meinem Sehen existiert [...], dann wäre die Anschauung selbst schon keine wahre Anschauung; sie hätte eine repräsentierende Funktion, und wir hätten den Fall 2). ${ }^{51}$

Husserl lehnt also die traditionelle Ansicht ab, nach der die Existenz (des Gegenstands) einer Vorstellung eine Beziehung von ihr zu einem Gegenstand implizieren würde, der nicht der Vorstellung innewohnt. Eine solche Interpretation der Existenzialurteile, die er dem zeitgenössischen Tübinger Logikprofessor Christoph von Sigwart zuschrieb, ist laut Husserl unzureichend, weil sie eine falsche Verdoppelung des gesehenen Schreibtischs mit sich bringt: ,Der Schreibtisch existiert nicht bloß als meine Anschauung, sondern auch unabhängig von ihr? Dann hätten wir eine doppelte Existenzbehauptung." (Hua XL, 31)

50 Z.B. Hua XXII, 107-108. Vgl. Schuhmann 2004, 107 ff.

51 Ibid. 
Author's accepted manuscript deposited in the Open Access repository (http://real.mtak.hu/) of the Hungarian Academy of Sciences (HAS) by the author under obligation imposed by the HAS, in accordance with the Copyright Transfer Agreement. Copyright 2015, All Rights Reserved by Springer Science+Business Media Dordrecht. Publishing on other websites is not covered by the exemption granted to the author by the Copyright Transfer Agreement. Scientific use only. Warning: There are discrepancies between this version and the final published one.

Published in Husserl Studies 31/2 (2015), pp. 95-121.

"The final publication is available at Springer via http://dx.doi.org/10.1007/s10743-014-9155-z"

Husserls Zurückweisung dieser Lösung ist einerseits sehr bemerkenswert, weil sich darin schon ein Grundprinzip seiner Phänomenologie bekundet: die Ablehnung einer supponierten Wirklichkeit hinter den Phänomenen. Wir haben gesehen, dass sich dieses Grundprinzip, obwohl nicht klar ausgearbeitet, schon Robert Zimmermanns Hume-Interpretation entnehmen lässt. Husserl wird sich konsequent daran halten, aber dessen Durchführung ist alles andere als trivial. ${ }^{52}$ In jener Ablehnung sieht sich Husserl von Brentanos Urteilstheorie unterstützt: „[a]ngenommen nun, die Existenzbehauptung beziehe sich auf das jetzt Vorgestellte, also auf die Anschauung unmittelbar, wie Brentano es will““ (32). Interessanterweise geht Husserl zugleich aber auf Distanz von Brentano: „diese Affirmation [ist] jedenfalls nicht immer da [...], wenn wir anschauen (wenigstens finden wir davon in innerer Erfahrung nichts)“ (32). Husserl hält also auch Brentanos Urteilstheorie für unzureichend, die - in ihrer einfachsten Form (ohne Berücksichtigung der Doppelurteile) - das Urteil als ein auf die Vorstellung aufbauendes Moment der Affirmation oder Ablehnung auffasst. Husserls eigene Lösung antizipiert hier schon die Erfüllungstheorie der Sechsten Untersuchung. Er erklärt sie an folgendem Beispiel: ,, Es ist Feuer!‘ [...] ,Feuer` für sich allein ist zunächst Ausdruck der repräsentierenden Vorstellung, das , ist ${ }^{`}$ führt zur Sache über, zum Entsprechenden und als Anschauung Seienden.“(33) Das Existenzialurteil besteht also weder in der Behauptung einer Beziehung der Vorstellung auf etwas Äußerliches (Sigwart) noch in dem Hinzutreten eines Moments $\mathrm{zu}$ der Vorstellung (Brentano), sondern in der spezifischen Form des Übergangs: „Expliziere ich mir die Bedeutung des Ausdrucks ,Es ist Feuer“, so würde ich vielleicht sagen: Wenn ich vor der Marienkirche stände, würde ich sie brennen sehen. Ich würde dann die der Vorstellung entsprechende Anschauung haben.“ (34) M.a.W., das von Brentano gesuchte Moment besteht eigentlich in der „eigentümliche[n] Verstärkung und Befriedigung beim Übergang vom Vorgestellten zum Angeschauten“ (34). Diese Analyse antizipiert besonders klar Husserls reife phänomenologische Position, weil er sogar auf die metaphysische Neutralität der Beschreibung des Übergangs hinweist: Die „entsprechende Anschauung [...] könnte auch [eine] Halluzination sein.“ “53 Es ist also die Aufgabe der Phänomenologie, die verschiedenen Leistungen des Bewusstseins

52 In einem anderen Text dieser Zeit versucht Husserl, diese Schwierigkeit durch die Unterscheidung zwischen phänomenalem und transzendentem Ding zu überwinden, wobei das letztere dem „Produkt einer auf die Dingvorstellungen des gemeinen Lebens bezogenen Reflexion“" entsprechen soll (Hua XXII, 111).

53 Husserls (spätere) Randbemerkung, Hua XL, 33 Anm. 2. 
Author's accepted manuscript deposited in the Open Access repository (http://real.mtak.hu/) of the Hungarian Academy of Sciences (HAS) by the author under obligation imposed by the HAS, in accordance with the Copyright Transfer Agreement. Copyright 2015, All Rights Reserved by Springer Science+Business Media Dordrecht. Publishing on other websites is not covered by the exemption granted to the author by the Copyright Transfer Agreement. Scientific use only. Warning: There are discrepancies between this version and the final published one.

Published in Husserl Studies 31/2 (2015), pp. 95-121.

"The final publication is available at Springer via http://dx.doi.org/10.1007/s10743-014-9155-z"

voraussetzungslos und mit wissenschaftlicher Strenge zu untersuchen, unabhängig davon, ob sich eine Bewusstseinsleistung im späteren Verlauf als wesentlich modifiziert herausstellen wird. ${ }^{54}$ Husserls partielle Bevorzugung der sigwartschen Lösung ist umso gewichtiger, als der oben erwähnte „Feuer“-Satz ursprünglich ein Beispiel Sigwarts war:

Drücke ich eine von mir gemachte Wahrnehmung in den Worten aus: das Schloss brennt, so ist mein Ausgangspunkt das Bild des brennenden Schlosses; in diesem erkenne ich die bekannte Gestalt des Gebäudes und die aus demselben schlagenden Flammen; indem ich diese beiden Elemente zuerst unterscheide und dann im Satze vereinige, beschreibe ich, was ich sah. ${ }^{55}$

In Husserls Text finden wir eine ziemlich ähnliche Paraphrase dieser Beschreibung:

Expliziere ich mir die Bedeutung des Ausdrucks „Es ist Feuer“, so würde ich vielleicht sagen: Wenn ich vor der Marienkirche stände, würde ich sie brennen sehen. Ich würde dann die der Vorstellung entsprechende Anschauung haben. Ich stelle also die eigentümliche Verstärkung und Befriedigung beim Übergang vom Vorgestellten zum Angeschauten vor. ${ }^{56}$

Das Kriterium für die Wahrheit des Satzes und dessen Ausdrucks ist schon bei Sigwart die Entsprechung mit der passenden Wahrnehmung, ${ }^{57}$ und Husserl hat dies mit der Einführung der Übergangssynthesis phänomenologisch weiter entwickelt. Das antizipiert bereits eindeutig die Sechste Untersuchung. ${ }^{58}$ Jedoch sollen wir uns vor einer allzu optimistischen Stellungnahme hüten, weil eine einfache Rückprojektion gerade die entwicklungsgeschichtlich relevanten Aspekte verdecken würde. Husserl hat sich nämlich in dem Text von 1893-94 ganz klar einer disjunktivistischen Theorie zugunsten Sigwarts verpflichtet:

Danach scheint jede Existenzbehauptung im letzten Grund vorauszusetzen das Verhältnis einer (repräsentierenden) Vorstellung zur Sache, aber nur logisch, nicht psychologisch. Sigwart hat Recht, wenn er für jedes Urteil eine Beziehung für erforderlich hält. Aber er hat Unrecht, wenn

54 Vgl. Hua XIX/1, 387 (A 353), bes. Anm. 1. Gerade diese Indifferenz gegenüber den Halluzinationen hob Dan Zahavi als Charakteristikum von Husserls metaphysischer Neutralität hervor $(2002,106)$, die aber in die Richtung der späteren transzendentalen Phänomenologie weist (im Sinne der zweiten Interpretationsmöglichkeit, 102).

55 Sigwart 1889, 26 (dieser Satz ist in Husserls Exemplar teilweise unterstrichen und mit einer Anstreichung am Rand versehen).

56 Hua XL, 34.

57 Vgl. Sigwart 1889, 81, 89 ff.

$58 \mathrm{Vgl}$. besonders Hua XIX/2, $566 \mathrm{ff}$. 
Author's accepted manuscript deposited in the Open Access repository (http://real.mtak.hu/) of the Hungarian Academy of Sciences (HAS) by the author under obligation imposed by the HAS, in accordance with the Copyright Transfer Agreement. Copyright 2015, All Rights Reserved by Springer Science+Business Media Dordrecht. Publishing on other websites is not covered by the exemption granted to the author by the Copyright Transfer Agreement. Scientific use only. Warning: There are discrepancies between this version and the final published one.

Published in Husserl Studies 31/2 (2015), pp. 95-121.

"The final publication is available at Springer via http://dx.doi.org/10.1007/s10743-014-9155-z"

er glaubt, dass dies in psychologischem Sinn gilt. $^{59}$

Husserl betont dies sogar gegenüber Brentano: „[Brentano] hat Unrecht, wenn er die logisch dem Urteil zugrunde liegende Beziehung auf einen Gegenstand leugnet“ (Hua XL, 36).

Zwei Aspekte sind hier bemerkenswert: Einerseits standen wichtige Teile seines phänomenologischen Instrumentariums Husserl schon 1893-94 zur Verfügung, die aber zugleich tief in der herbartianischen Tradition verwurzelt sind. ${ }^{60}$ Andererseits sucht Husserl in seiner geteilten Loyalität Existenzbehauptung mit Hilfe von Sigwart auch „logisch“ zu verankern. Dies wird sich ändern, wenn Husserl sich in die schulinterne Diskussion der Brentano-Schüler über Intentionalität einschaltet - aber in welche Richtung?

\section{b) Husserls Auseinandersetzung mit der Intentionalitätsdebatte der Brentano-Schule}

Es gibt ein umfangreiches Manuskript vom Sommer 1894, das Husserl selbst als „Reaktion gegen Twardowski“ bezeichnet hat, und das als das entscheidende erste Dokument seiner Auseinandersetzung mit der Diskussion der Brentano-Schule betrachtet wurde. Denn dieses führte Husserl schließlich zu seinem eigenen Intentionalitätsverständnis. ${ }^{61}$ Diese Diskussion wurde vor allem von Brentanos nicht-orthodoxen Schülern geführt. Alois Höfler, einer seiner eigenständigen Schüler, die Bolzanos Bedeutung anscheinend unabhängig von Brentano entdeckt hatten, hat schon in seinem 1890 (unter nomineller Mitwirkung von Meinong) verfassten Logik-Lehrbuch das Problem der Doppeldeutigkeit des Vorstellungsgegenstands deutlich hervorgehoben: „Die Wörter ,Gegenstand“ und ,Object' werden in zweierlei Sinn gebraucht: einerseits für dasjenige an sich Bestehende, [...] Reale [...], worauf sich unser Vorstellen und Urtheilen gleichsam richtet, andererseits für das , in ' uns bestehende psychische [...] ,Bild 'von jenem Realen“" (Höfler 1890, 7). Zugleich hat Höfler die Bedeutung des paradigmatischen Falls der gegenstandslosen Vorstellungen,

59 Hua XL, 35.

60 Ein herbartianischer Einfluss wurde schon vermutet (Hua XXII, liii Anm. 3, Schuhmann 2004, 113), aus Husserls Anknüpfung an Sigwart können wir aber einen konkreten Vermittlungsmechanismus entnehmen.

61 Der brentanosche Begriff der Intentionalität wurde von Husserl „offenbar erstmals im Sommer 1894, und zwar in dem gegen Twardowski geschriebenen Manuskript“ verwendet (Schuhmann 2004, 111 Anm. 32, vgl. 120); ,der Husserlsche Begriff der Intentionalität [ist] [...] nicht so sehr von Brentano als vielmehr von Twardowski her zu verstehen“ (117). Vgl. auch Ierna 2006, 57-58. 
Author's accepted manuscript deposited in the Open Access repository (http://real.mtak.hu/) of the Hungarian Academy of Sciences (HAS) by the author under obligation imposed by the HAS, in accordance with the Copyright Transfer Agreement. Copyright 2015, All Rights Reserved by Springer Science+Business Media Dordrecht. Publishing on other websites is not covered by the exemption granted to the author by the Copyright Transfer Agreement. Scientific use only. Warning: There are discrepancies between this version and the final published one.

Published in Husserl Studies 31/2 (2015), pp. 95-121.

"The final publication is available at Springer via http://dx.doi.org/10.1007/s10743-014-9155-z"

deren logischer Umfang gleich Null ist, ${ }^{62}$ klar erkannt. Twardowskis Habilitationsschrift von 1894 knüpft dann explizit an dieser Frage an (Twardowski 1894, 4 ff.), die er als Kollision zwischen Brentanos und Bolzanos Ansichten verstanden hat (20 ff.).

In dem erhaltenen Teil jenes Manuskripts hat Husserl offenbar an dieser Problemstellung angeknüpft: „Jeder Vorstellung entspricht ein Gegenstand. Andererseits [gibt es...], mit Bolzano zu sprechen, ,gegenstandslose Vorstellungen““ (IG 142). Für Husserl liegt die Schwierigkeit darin, dass eine Verdoppelung des Vorstellungsgegenstands bzw. eine disjunktive Theorie, welche die paradoxe Gegenüberstellung der beiden Thesen leicht auflösen könnte, in der Erfahrung schlicht nicht aufzuweisen ist. Die konträren Thesen bedeuten gerade, „daß jeweils derselbe Gegenstand, der vorgestellt ist, existiert bzw. nicht existiert“ (144), oder, wie Husserl es an folgendem anschaulichen Beispiel zeigt: „Dasselbe Berlin, das ich vorstelle, existiert auch, und dasselbe würde nicht mehr existieren, bräche ein Strafgericht ein wie bei Sodom und Gomorrha“ (144).

Husserls Beharren auf der allgemeinen und einheitlichen Gültigkeit der Intentionalität weist in die Richtung seiner späteren Phänomenologie. Die Frage ist nur, wie er jene in dem Text von $1894 \mathrm{zu}$ implementieren vermag. Husserls Position in diesem Text hat die Interpreten irritiert, was vielleicht damit zusammenhängt, dass sein Denkhintergrund in der Forschung bislang unberücksichtigt blieb, ${ }^{63}$ obwohl Husserl selbst einige Quellen benannt hat in einer Anmerkung, die wohl schon zu einer früheren Fassung des Texts gehört. ${ }^{64}$ Dort weist er auf eine Stelle in Sigwarts Logik und auf eine Parallele in dessen Abhandlung über die Impersonalien hin, wo Sigwart genau auf dieselbe Argumentation zurückgreift. Sigwarts Gegner war Franz Kern (1830-1894), ein Berliner Gymnasialdirektor, dessen Verbesserungsvorschläge zur deutschen Grammatik lebhafte Resonanz bei den Zeitgenossen hervorgerufen haben. Bei seinen Untersuchungen der Funktionen der Kopula war Kern auf die Funktion gestoßen, ,dass das Sein von etwas ausgesagt werden kann und sehr oft ausgesagt wird, was ausser uns gar nicht existiert, oft überhaupt nicht existiert hat, vielleicht so gar nicht existieren kann. ${ }^{\text {"65 }}$ Kerns Hinweis ist insofern berechtigt, als in komplexen fiktiven Welten,

62 Höfler 1890, 26, 30-31. Laut Husserls Lesespuren konnte ihm Höfler hierbei offenbar wenig Neues bieten.

63 Sogar Rollinger geht dem Denkhintergrund von Husserls Position nicht nach (vgl. 1999, 149 ff.).

64 Ms. K I 56 / 10a, vgl. Hua XXII, 457.

65 Kern 1888, 91. - Sigwarts Referenzen sind ungenau. Husserl kannte das Buch wohl nur durch Sigwarts Bericht; die Namensangabe bzw. die bibliografischen Angaben in Hua XXII sind mangelhaft, und Schuhmanns Index Nominum identifiziert ihn fälschlicherweise mit Berthold von Kern. 
Author's accepted manuscript deposited in the Open Access repository (http://real.mtak.hu/) of the Hungarian Academy of Sciences (HAS) by the author under obligation imposed by the HAS, in accordance with the Copyright Transfer Agreement. Copyright 2015, All Rights Reserved by Springer Science+Business Media Dordrecht. Publishing on other websites is not covered by the exemption granted to the author by the Copyright Transfer Agreement. Scientific use only. Warning: There are discrepancies between this version and the final published one.

Published in Husserl Studies 31/2 (2015), pp. 95-121.

"The final publication is available at Springer via http://dx.doi.org/10.1007/s10743-014-9155-z"

z.B. in der Welt des Mythos, auch Aussagen wie „Apollon ist der Beschützer der Troer“ sinnvoll sind (Kern 1888, 91). Kern zieht daraus aber den naiven Schluss, dass diese eine Realität ,in den Vorstellungen des Redenden“ bezeichneten (92). Außerdem wendet Kern dies auch auf kopulative Sätze über logisch unmögliche Konstruktionen an, wie „ein viereckiger Kreis ist ein Widerspruch“ (ibid.). Die Schwäche seines Ansatzes kommt klar zum Vorschein, wenn er sagt: „,so existiert jenes Eisen und dieser Kreis nur in meiner Vorstellung[,] und zwar mit der von mir erkannten und ausgesprochenen Eigenschaft, ein Unding oder ein Widerspruch zu sein, [ist] also in einer von mir unabhängigen Wirklichkeit unmöglich anzutreffen“ (ibid.). Dagegen wendet Sigwart völlig zu Recht ein, dass „Widersprechendes [...] nicht bloss in der von mir unabhängigen Wirklichkeit, sondern auch in meinen Gedanken unmöglich“ ist (Sigwart 1889, 123 Anm.). Was Kern bei seiner Interpretation völlig übersieht, ist gerade der Unterschied zwischen der Vorstellung als einem immanenten mentalen Akt und dem Gegenstand des Vorstellens, den die schulinterne Diskussion explizieren und weiterentwickeln wollte. Für Husserl diente die Behauptung Kerns, die dieser wohl von Sigwart übernommen hat, anscheinend als plastische Illustration für eine widersprüchliche Position, die er später sogar Meinong zuschrieb. So notierte Husserl neben den Literaturangaben von Sigwart: „Wonach Kern allen Ernstes die Existenz des Absurden in der Vorstellung behauptet hat" (Hua XXII, 457). Dieselbe Bemerkung mit denselben Literaturangaben finden wir auch in einer Notiz, die er dem Text beigelegt hat. ${ }^{66}$

Aber dieser Hinweis ist keineswegs nur eine unbedeutende Veranschaulichung eines trivialen Fehlers, sondern Sigwart knüpft hier an eine Diskussion an, die explizit Herbart hervorrief und seitdem, wie wir oben bei Lindner gesehen haben, fester Bestandteil der herbartianischen Lehrbücher war. Im Lehrbuch zur Einleitung in die Philosophie vertrat Herbart, im Anschluss an die Definition des Urteils, das er traditionell als logische Verknüpfung zweier Begriffe deutete, die These, dass der Subjektbegriff des Urteils „keineswegs absolut, sondern nur hypothetisch [...] aufgestellt“" wird (Herbart 1850, 92). Diese Aussage richtet sich eigentlich gegen den ontologischen Import der kopulativen Sätze, was er sogleich an folgendem Beispiel des viereckigen Zirkels illustriert:

66 Ms. K I 56/9a, siehe Hua XXII, 457-458. 
Author's accepted manuscript deposited in the Open Access repository (http://real.mtak.hu/) of the Hungarian Academy of Sciences (HAS) by the author under obligation imposed by the HAS, in accordance with the Copyright Transfer Agreement. Copyright 2015, All Rights Reserved by Springer Science+Business Media Dordrecht. Publishing on other websites is not covered by the exemption granted to the author by the Copyright Transfer Agreement. Scientific use only. Warning: There are discrepancies between this version and the final published one.

Published in Husserl Studies 31/2 (2015), pp. 95-121.

"The final publication is available at Springer via http://dx.doi.org/10.1007/s10743-014-9155-z"

Das Urtheil, $A$ ist $B,[\ldots]$ enthält keineswegs die gewöhnlich hinzugedachte, aber ganz fremdartige, Behauptung, dass $A$ sei; denn von $A$ für sich allein, und von seinem Dasein, seiner Gültigkeit ist da keine Rede [...]. Das Urtheil: der viereckige Cirkel ist unmöglich, schliesst gewiss nicht den Gedanken in sich, der viereckige Cirkel sei vorhanden; sondern es bedeutet, wenn ein viereckiger Cirkel gedacht wird, so muss der Begriff der Unmöglichkeit hinzugedacht werden. ${ }^{67}$

$\mathrm{Zu}$ Husserls Zeit hat dieser Ansatz schon eine breite Diskussion ausgelöst, zu der die etablierten Gesamtdarstellungen der Logik Stellung genommen haben, wie z.B. Friedrich Ueberwegs System der Logik, wo die entsprechende Stelle von Husserl annotiert wurde. ${ }^{68}$ Husserl hat aber keineswegs Ueberweg benötigt, denn eine der klarsten und ausführlichsten Darstellungen der herbartianischen Position findet sich in Lindners Lehrbuch, das Husserl aus seiner Schulzeit kannte:

Der letztere [metaphysische] Sinn ist nun der Copula , ist ${ }^{\star}$ in einem Urtheile trotz der entgegenstehenden Meinungen mancher Logiker im allgemeinen nicht zuzuerkennen, und aus dem Urtheile , $\mathrm{S}$ ist $\mathrm{P}^{\prime}$ folgt noch keineswegs, daß $\mathrm{S}$ ist. Aus den Urtheilen: ,Neptun ist ein Meergott', ,Chimäre ist ein Phantasiegebilde', [...] ,wahre Freunde sind zu schätzen', folgt keineswegs, daß Neptun, die Chimäre [...] oder daß wahre Freunde existieren, sondern nur, daß, wenn man diese Begriffe denkt, man auch die von ihnen in den obigen Urtheilen prädicierten Begriffe mitzudenken habe. Daraus folgt, daß auch bei dem kategorischen Urtheile der Sinn der Aussage ein hypothetischer ist. ${ }^{69}$

Ueberwegs Stellungnahme dazu, die auch in der von Husserl direkt zitierten Anmerkung bei

67 Herbart 1850, 92-93. Damit dürfte Herbart die Sätze über den viereckigen Kreis als spezifisches Beispiel für das Problem des ontologischen Imports der Aussagen eingeführt haben (zuerst Herbart 1813, 37). Der viereckige Zirkel war in der neuzeitlichen Diskussion natürlich schon bekannt und lässt sich chronologisch mindestens auf den Cartesianer Johann Clauberg (1622-1665) zurückzuführen, aber nur als Beispiel für einen in sich widersprüchlichen Begriff (contradictio in adiecto) und für das Problem, ihn zu denken (wie bei Leibniz, Kant, und sogar bei Herbart selbst an anderen Stellen, siehe z.B. Herbart 1813, 24, 167). Für Bolzano waren die sog. widersprechenden Vorstellungen (Bolzano 1837, Bd. I, 316 ff.), die die wichtigste Art gegenstandsloser Vorstellungen bilden und irreführend auch „imaginäre Vorstellungen“ genannt werden, selbstverständlich auch kein noch unbetretenes Neuland. Seinen eigenen Hauptverdienst sah Bolzano aber eher darin, mit seiner konsequenten Unterscheidung von Inhalt, Umfang und subjektiver Realisation der Vorstellungen die Missverständnisse ausräumen zu können. Husserls Lesespuren in seinem Exemplar der Wissenschaftslehre legen auch nicht nahe, dass ihn Bolzano hier wesentlich überraschen konnte (er interessierte sich eher für spezifische Momente, z.B. für die Möglichkeit unvollständiger sinnlicher Begleitbilder für widersprechende Vorstellungen, s. Bd. II, 321) - was uns in Anbetracht des oben Dargelegten gar nicht verwundert.

68 Ueberweg 1882, 204-205. An der oben zitierten Stelle knüpft auch Sigwart an diese Diskussion an.

69 Lindner 1872, 40. 
Author's accepted manuscript deposited in the Open Access repository (http://real.mtak.hu/) of the Hungarian Academy of Sciences (HAS) by the author under obligation imposed by the HAS, in accordance with the Copyright Transfer Agreement. Copyright 2015, All Rights Reserved by Springer Science+Business Media Dordrecht. Publishing on other websites is not covered by the exemption granted to the author by the Copyright Transfer Agreement. Scientific use only. Warning: There are discrepancies between this version and the final published one.

Published in Husserl Studies 31/2 (2015), pp. 95-121.

"The final publication is available at Springer via http://dx.doi.org/10.1007/s10743-014-9155-z"

Sigwart angesprochen wurde, hat Husserl in seinem Exemplar annotiert. Ueberweg argumentiert für den ontologischen Import der kopulativen Sätze: „Ein Satz, wie: wahre Freunde sind zu schätzen, beruht auf der Voraussetzung, dass es solche gebe“ (Ueberweg 1882, 205). Er versucht aber, die Opposition zu Herbart abzumildern, indem er zugleich folgende Vermittlung ergänzt: „wer die Voraussetzung nicht annehmen will, muss jenen Sätzen die Clauseln beifügen, wodurch sie zu hypothetischen werden“ (ibid.). Laut Ueberweg lässt sich eine solche Klausel auch implizit durch den Verbmodus ausdrücken oder sie kann sich sogar aus dem Zusammenhang ergeben:

Nur wenn der Zusammenhang des Ganzen (wie in einem Roman) oder der bekannte Sinn eines Wortes (wie Zeus, Sphinx, Chimäre etc.) auf eine bloss fingierte Wirklichkeit oder auch auf eine blosse Namenerklärung hinweist, ist eine derartige Clausel entbehrlich. ${ }^{70}$

Gerade diese Lösung taucht später bei Husserl als dessen eigene Antwort auf:

Die Vorstellungen ,Zeus ‘ und ,der oberste der olympischen Götter` haben denselben intentionalen Gegenstand, d.h. Zeus ist der oberste der olympischen Götter - nach der griechischen Mythologie. Wir lassen diesen Zusatz in der Regel weg und vermissen ihn nicht, wo er fehlt: Es ist selbstverständlich, daß, wer über mythische Objekte urteilt, sich auf den Boden des Mythus [sic!] stellt, ohne sich ihn doch wahrhaft zu eigen zu machen. ${ }^{11}$

Eine solche Lösung benötigt Husserl für die Interpretation des brentanoschen Teils des ursprünglichen Paradoxons, da er dessen anderen Teil schon uneingeschränkt übernommen hat: „Nicht jede Vorstellung hat einen Gegenstand“ (IG 150).

Die Beschäftigung mit der schulinternen Diskussion über die Intentionalität führte Husserl also keineswegs zu der Aneignung von Brentanos Position. Im Gegenteil: Wenn man nicht Husserls Auseinandersetzung mit den Brentano-Schülern, sondern seine eigene Antwort betrachtet, wird klar, dass er sich bei diesem Teil des ursprünglichen Paradoxons im Grunde genommen der herbartianischen Position angeschlossen hat, wonach die kategorischen Aussagen letztlich auf eine hypothetische Struktur zurückführen. Denn die ,intentionalen“ Gegenstände hat Husserl auf diese Weise interpretiert: „Fragt man nun, was es in dem hier fraglichen Sinne heißt, zwei Vorstellungen stellten [...] denselben Gegenstand vor, so sieht man sofort, daß hier nur Identität gemeint sein kann

70 Ueberweg 1882, 205.

71 IG 151. 
Author's accepted manuscript deposited in the Open Access repository (http://real.mtak.hu/) of the Hungarian Academy of Sciences (HAS) by the author under obligation imposed by the HAS, in accordance with the Copyright Transfer Agreement. Copyright 2015, All Rights Reserved by Springer Science+Business Media Dordrecht. Publishing on other websites is not covered by the exemption granted to the author by the Copyright Transfer Agreement. Scientific use only. Warning: There are discrepancies between this version and the final published one.

Published in Husserl Studies 31/2 (2015), pp. 95-121.

"The final publication is available at Springer via http://dx.doi.org/10.1007/s10743-014-9155-z"

unter einer Hypothese der Existenz. ${ }^{\text {(72 }}$

Husserls Präferenz der herbartianische Auffassung ist umso interessanter, weil Bolzano sich in dieser Diskussion ausdrücklich gegen Herbart positioniert hat. Schon bei den Anschauungen insistiert Bolzano darauf (Bolzano 1837, Bd. I, 362), dass jeder Anschauung ein existierender Gegenstand entspreche (was Husserl in seinem Exemplar mit einem Fragezeichen versehen hat), und vertritt später ausdrücklich die These, dass ,die sogenannte Subjektvorstellung [...] in allen wahren Sätzen eine eigentliche Gegenstandsvorstellung“ sein müsse (Bd. II, 328). Gegenstandslose Vorstellungen dürfen also nicht als Subjekte in wahren Sätzen fungieren. ${ }^{73}$ Bolzano stand also wenn man die Wissenschaftslehre genauer betrachtet - Brentanos These von der universalen Geltung der Intentionalität näher, als man auf Grund seiner anfänglichen Definitionen vermuten würde. Umso deutlicher ist Husserls Stellungnahme gegen diese Position. ${ }^{74}$

\section{c) Husserls Position im Kontext der Weiterentwicklung seiner Protophänomenologie}

Husserls eigenartige Positionierung hat verschiedenste Urteile ausgelöst. B. Rang sah darin „eine

72 Fassung erster Hand nach Ms. K I 56/15a, 17a.

73 Etwaige sich anbietende Gegenbeispiele versucht Bolzano durch die Hervorhebung der tieferen logischen Strukturen zu entkräften. Der Satz „der viereckige Zirkel ist unmöglich“ (Bolzano 1837, Bd. II, 404) verändert sich bei Bolzano in einen kategorischen Satz über die Vorstellung eines viereckigen Zirkels, von der die Eigenschaft der Gegenstandlosigkeit prädiziert wird. Er fragt jedoch nicht, wie der Satz „,der viereckige Zirkel ist viereckig“ zu verstehen sei. Unter Berücksichtigung eines früheren Paragrafen (siehe Bd. I, 513-515; in Husserls Exemplar weist auch dieser Paragraf Lesespuren auf), in dem er die Verhältnisse gegenstandsloser Vorstellungen mit Hilfe einer rudimentären Quantor-Theorie interpretiert, wäre diese Schwierigkeit für Bolzano prinzipiell nicht unüberwindbar. Der Preis, den er dafür zahlen müsste, bestünde darin, dass, während die erste Umwandlung lediglich eine Klarstellung der eigentlichen Satzstruktur bedeutet, die zweite bekanntlich schon einen wesentlichen Umbau des Satzes mit sich bringt.

74 Karl Schuhmann (2004, 129, in Anlehnung an Bernhard Rang, Hua XXII, xlv ff.) weist auf eine Anmerkung Brentanos hin, wo dieser, gegen J. St. Mill, Sätze wie „ein Centaur ist eine Erfindung der Poeten“ diskutiert (Brentano 1874, 286 Anm. 1). Allerdings täuscht diese Ähnlichkeit, da Brentano diese Frage letztlich mit Hilfe der Unterscheidung von attributivem (determinierendem) und modifikativem Gebrauch der Adjektive löst (,gelehrter Mensch“vs. „toter Mensch“, 288), was bekanntlich den Grundpfeiler von Twardowskis Habilitationsschrift bildete. Dieser suchte damit die Annahme der Existenz miteinander unverträglicher Gegenstände zu entkräften: „Durch den Zusatz: [existiert] als Vorstellungsgegenstand, wird die Bedeutung des Ausdrucks Existenz modificiert“" (Twardowski 1894, 24-25, vgl. 13 ff.). Diese Lösung hat Husserl jedoch ausdrücklich abgelehnt: „Es wird auch unverständlich, was die Modifikation hier eigentlich will und meint“" (IG 146). Falls gelegentlich eine (sprachliche) Ähnlichkeit zwischen Twardowski und Husserl auszumachen ist (z.B. IG 150), ist sie andererseits dadurch zu erklären, dass Husserl „Modifikation“ mit einem ganz neuen Inhalt zu erfüllen beabsichtigte - den er aber von Herbart ererbt hat. Im Übrigen scheint auch Brentano 1906 bewusst gewesen zu sein, dass der bloße Hinweis auf „Modifikation“ in der Luft schweben bleibt, s. Brentano 1977, 160. 
Author's accepted manuscript deposited in the Open Access repository (http://real.mtak.hu/) of the Hungarian Academy of Sciences (HAS) by the author under obligation imposed by the HAS, in accordance with the Copyright Transfer Agreement. Copyright 2015, All Rights Reserved by Springer Science+Business Media Dordrecht. Publishing on other websites is not covered by the exemption granted to the author by the Copyright Transfer Agreement. Scientific use only. Warning: There are discrepancies between this version and the final published one.

Published in Husserl Studies 31/2 (2015), pp. 95-121.

"The final publication is available at Springer via http://dx.doi.org/10.1007/s10743-014-9155-z"

eigentümliche epoché، (Hua XXII, xxxvii) und sogar eine ,überraschend klare Vorwegnahme des Gedankens von der Einzigkeit der Welt, der zum Leitfaden des Weltproblems der Husserlschen Spätphilosophie geworden ist“" (xli), während R. Rollinger Husserls Lösung gerade dafür preist, dass sie dem späteren idealistischen Standpunkt der Ideen vorzuziehen ist und zugleich den Platonismus vermeidet (Rollinger 1999, 151). K. Schuhmann hat Husserls „denkökonomischen Kunstgriff“" sogar einmal mit Thomas Kuhns Begriff des Paradigmas verglichen (Schuhmann 2004, 127-128). Diese ganz unterschiedlichen Deutungen zeigen sehr klar, dass erst der oben vorgenommene Offenlegungsversuch des historisch-biografischen Denkhintergrunds den Schlüssel für das Verständnis dieses Texts bietet.

Schon bei der Definition des Begriffs wies Lindner auf die Möglichkeit der „bloße[n] Gedankendinge“ hin, die sich „sich so gut wie Naturkörper definieren, classificiren, vergleichen“ etc. lassen (siehe Abschnitt 4.a) ). Diese Tradition scheint in der Tat richtunggebend für Husserl gewesen zu sein. Zugleich birgt sie aber auch Unklarheiten in sich, indem sie solche wichtige Definitionen, wie die des Begriffs, nicht hinreichend ausarbeitet. Husserl hat sich damit auseinandergesetzt (siehe Abschnitt 4.b)), und die Vermutung liegt nahe, dass er gerade wegen dieser Unklarheiten Bolzano zur Unterstützung heranzog. Husserls Begegnung mit ihm ist, wie diejenige mit Lotze, in mehreren Stufen erfolgt, ${ }^{75}$ und im Text findet sich möglicherweise Husserls erste Verwendung des „Bolzanoschen Terminus objektive Vorstellung“ (IG 166).

Der im Vorangegangenen freigelegte herbartianische Hintergrund bildet aber nicht bloß eine Vorgeschichte der Wende Husserls zu Bolzano. Husserl hat später wiederholt gegen eine voreilige Identifikation von Bolzanos Sätzen an sich und der objektiven Seite seines Standpunkts gewarnt und betont, „dass Bolzanos Erkenntnistheorie auf dem Boden eines extremen Empirismus steht.“ ${ }^{76}$ Dies ist in dieser Form unhaltbar ${ }^{77}$ und kann allenfalls als eine Übertreibung verstanden werden, mit

75 Husserl selbst berichtete, dass er zuerst „,auf Bolzano als Mathematiker“ aufmerksam wurde (Hua XX/1, 297 Anm. 2), und sein Studium der ,längstvergessene[n] Wissenschaftslehre“ dürfte - wohl später (vielleicht gerade ,in d[en] [18]90-er Jahre[n]“", BW VII, 97) - durch eine zufällige Entdeckung in einem Antiquariat ausgelöst worden sein (vgl. Schuhmann 1977, 463), wohl vorbereitet von Benno Kerrys Aufsatzreihe (1885-1891), durch die Husserl auf das Buch aufmerksam werden musste (vgl. Künne 2008, 326 ff., 349 ff.).

76 Hua XX/1, 308, siehe auch Hua XXII, 156 ff.; BW I 29, 39; VII, 98.

77 Husserls Belegstelle ist Bolzano 1837, 244 (in Husserls Exemplar markiert und zitiert: Hua XX/1, 309). Dagegen bemerkt aber Bolzano: Was „durch Gründe der Vernunft gewiß ist, kann auch durch keine scheinbar entgegenstehenden Erfahrungen widerlegt werden“ (Bolzano 1837, 52). Bei seiner Belegstelle verkennt Husserl offenbar den Gedankengang von Bolzanos gegen Kant gerichteter Argumentation: Bolzano geht es da darum, dass 
Author's accepted manuscript deposited in the Open Access repository (http://real.mtak.hu/) of the Hungarian Academy of Sciences (HAS) by the author under obligation imposed by the HAS, in accordance with the Copyright Transfer Agreement. Copyright 2015, All Rights Reserved by Springer Science+Business Media Dordrecht. Publishing on other websites is not covered by the exemption granted to the author by the Copyright Transfer Agreement. Scientific use only. Warning: There are discrepancies between this version and the final published one.

Published in Husserl Studies 31/2 (2015), pp. 95-121.

"The final publication is available at Springer via http://dx.doi.org/10.1007/s10743-014-9155-z"

deren Hilfe Husserl seine Distanz zu Bolzano markieren will: Bolzano „Elementarlehre“ eignet sich nicht zum Zwecke einer ,erkenntnistheoretischen Aufklärung der reinen Logik“, wie Husserl die Hauptleistung der Logischen Untersuchungen im kurzen Rückblick bezeichnet hat (Hua XIX/2, 783). Dies zeigt sich z.B. besonders prägnant darin, dass Bolzano die „Lebhaftigkeit“ und andere Unterschiede der subjektiven Vorstellungen (der „Erscheinungen in unserem Gemüthe“) für irrelevant erachtete und nicht $\mathrm{zu}$ den objektiven Vorstellungen rechnete, die den Stoff der subjektiven Vorstellungen bilden (Bolzano 1837, Bd. I, 217-218). Trotz aller offenbaren Ähnlichkeit der Konzeptionen Bolzanos und Husserls (vgl. Künne 2011, 79-80), ermöglicht diese Entscheidung Bolzanos kaum die begriffliche Ausprägung des bedeutungsmäßigen Wesens und des Moments der Fülle ermöglicht, welche die Grundpfeiler der erkenntnistheoretischen Aufklärung der Logik in Husserls Sechsten Logischen Untersuchung bilden. Es ist wohl kein Zufall, dass wir gerade in diesem Zusammenhang Einflüsse entdeckt haben (siehe Abschnitt 5.a) ), die weder Brentano noch Bolzano zuzuschreiben waren. Und wenn Husserl kurz nach der Veröffentlichung der Logischen Untersuchungen meinte, „Bolzano selbst zeigt nicht mit der entferntesten Andeutung, daß diese phänomenologischen Beziehungen zwischen Bedeutung, Bedeutungsmoment und vollem Akt des Bedeutens von ihm bemerkt worden sein“ (Hua XXII, 157), können wir die philosophische Tradition, die wir oben bei Lindner und Zimmermann verortet und in Husserls späterer Auseinandersetzung mit der Brentano-Schule weiterverfolgt haben, nicht nur als ein historisches Relikt, sondern als ein Modell betrachten, das sich auch für das Verständnis von Husserls Position nach seiner expliziten Beschäftigung mit Bolzano in den späten 1890er Jahren als hilfreich erweisen könnte: Anstatt von einem vermeintlichen Platonismus auszugehen, ist Husserls Denkentwicklung in den Jahren unmittelbar vor den Logischen Untersuchungen offenbar eher als ein Versuch zu verstehen, neben der psychologischen Beschreibungsebene einen objektiven Bedeutungsgehalt zu

sich bei den Sätzen der reinen Logik und reinen Naturwissenschaft „,nicht leicht ein Irrthum einschleichen oder erhalten“ kann (244), und ob diese De-facto-Zuverlässigkeit ein Beweis für deren Analytizität sei. Weil für Bolzano solche Sätze aber synthetisch sind (vgl. 239 ff.), meint er, dass diese Zuverlässigkeit aus anderen Gründen folge (schon früh: Bolzano 1810, 150), und dass wir uns durch „Versuche“ und „Prüfung seiner Ableitungsart“ der „Richtigkeit eines Satzes“" vergewissern könnten (309). Husserl, der die Wissenschaftslehre - wie er selbst konzediert hat - „,mit Hilfe ihres reichhaltigen Index“ benutzte (Hua XX/1, 297 Anm. 2), hat wohl übersehen, dass die Ableitbarkeit (einer von Bolzanos drei spezifischen Konsequenzbegriffen, zu dem Husserl in seinem Exemplar eine Randbemerkung hinzugefügt hat, die seine Unwissenheit nahelegt) auch auf nicht-logischen Vorstellungen beruhen kann. M. a. W., mit den „Versuche[n]“ und der „Ableitbarkeit“ will Bolzano betonen, dass es sich bei diesen Sätzen gerade nicht um rein analytische Wahrheiten handelt. 
etablieren und die daraus resultierenden Probleme - wie das Problem der gegenstandslosen Vorstellungen - zu lösen. Hierbei hat Husserl sowohl in concreto als auch im Allgemeinen aus dem Erbe geschöpft, das er sich in seiner Schul- und Studienzeit außerhalb der Brentano-Schule zu eigen gemacht hat.

\section{Ausblick: Der genauere Kontext von Husserls Frühphilosophie}

In der deutschen Philosophie der vorletzten Jahrhundertwende galt Husserl zweifellos als Quereinsteiger, und zwar weniger wegen seiner naturwissenschaftlichen Ausbildung (denn, wie hier gezeigt wurde, Husserl verfügte über eine solide Grundausbildung in der Philosophie), sondern, weil er aus einem Land kam, dessen philosophische Entwicklung durch Ausbleiben einer ungehinderten Rezeption von Kant und dem Deutschen Idealismus einen anderen Kurs eingeschlagen hat als der nördliche Teil des deutschen Sprachraums. Diese Differenz wurde aber bisher stets auf Husserls Zugehörigkeit zur Brentano-Schule zurückgeführt (wobei zweifellos seine Selbstcharakterisierungen eine maßgebliche Rolle gespielt haben). In meinem Beitrag habe ich versucht, Quellen von Husserls philosophischer Ausbildung offenzulegen, die nicht direkt der Brentano-Schule zugeschrieben werden können. Diese erwiesen sich nicht als eine direkte Vermittlung von Bolzano, sondern haben Husserl mit einer logischen Tradition bekannt gemacht, die seine spätere Auseinandersetzung mit der Brentano-Schule über die Intentionalität nachweislich beeinflusst hat. Daran manifestiert sich ein Denkhintergrund, der sich nicht direkt auf Brentano oder Bolzano reduzieren lässt. Die Berücksichtigung dieses bislang weniger untersuchten Hintergrundes, der sich in häufigen Hinweisen auf inzwischen vergessene zeitgenössische Logiker und ebenso in den Annotationen seiner Bücher definitiv zeigt, bildet also ein wesentlicher Aspekt einer besser fundierten historischen Kontextualisierung der Entstehung von Husserls Philosophie.

\section{Danksagung}

Für wertvolle inhaltliche und formale Hinweise bin ich Prof. Dr. Dieter Lohmar (Köln), Dr. Dirk 
Fonfara (Heidelberg/Köln), ferner der Herausgeberin und einem anonymen Gutachter dieser Zeitschrift zu besonderem Dank verpflichtet. Prof. Dr. Ullrich Melle (Leuven) danke ich für die Erlaubnis, aus den unveröffentlichten Manuskripten Edmund Husserl zitieren zu dürfen. Die Wiener Archivforschungen wurden 2012 mithilfe des „Collegium Hungaricum“ Stipendiums des Ungarischen Staats durchgeführt. Affiliation während der abschließenden Phase der Manuskriptvorbereitung: Institut für Philosophie der Ungarischen Akademie der Wissenschaften, gefördert durch das Hungarian Scientific Research Fund (OTKA)-Forschungsprojekt Nr. PD105101.

\section{Unveröffentlichte Quellen (Ms.)}

Archiv des Kunsthistorischen Museums, Wien (KHM): IV 64.

Husserl-Archiv: K I 56, K I 59, K I 61, X III 1

Universitätsarchiv Wien (UA Wien): Nationalen PHIL, PHIL Rig Prot PN 268.

\section{Abkürzungen}

BBGA I 11/1 Bernard Bolzano: Wissenschaftslehre $\S \S 1-45$. Hrsg. von Jan Berg. Stuttgart-Bad Cannstatt: Frommann-Holzboog, 1985.

BBGA IIB 16/1 Bernard Bolzano: Philosophische Tagebücher 1811-1817. Erster Teil. Hrsg. von Jan Berg. Stuttgart-Bad Cannstatt: Frommann-Holzboog, 1981.

BBGA III 3/2 Bernard Bolzano: Briefe an František Přihonský 1836-1845. Hrsg. von Jan Berg. Stuttgart-Bad Cannstatt: Frommann-Holzboog, 2005.

BBGA III 3/3 Bernard Bolzano: Briefe an František Přihonský 1846-1848. Hrsg. von Jan Berg. Stuttgart-Bad Cannstatt: Frommann-Holzboog, 2005.

BBGA E 1 Eduard Winter: Bernard Bolzano. Ein Lebensbild. Stuttgart-Bad Cannstatt: Frommann-Holzboog, 1969.

BW I Edmund Husserl: Briefwechsel. Die Brentanoschule. Hrsg. von Elisabeth 
BW IV

BW VII

BW VIII

BW IX

Hua XIX/1

Hua XIX/2

Hua XX/1

Hua XXII

Hua XXX

Hua XL

IG

Schuhmann - Karl Schuhmann. Dordrecht: Kluwer AP, 1994.

Edmund Husserl: Briefwechsel. Die Freiburger Schüler. Hrsg. von Elisabeth Schuhmann - Karl Schuhmann. Dordrecht: Kluwer AP, 1994.

Edmund Husserl: Briefwechsel. Wissenschaftlerkorrespondenz. Hrsg. von Elisabeth Schuhmann - Karl Schuhmann. Dordrecht: Kluwer AP, 1994.

Edmund Husserl: Briefwechsel. Institutionelle Schreiben. Hrsg. von Elisabeth Schuhmann - Karl Schuhmann. Dordrecht: Kluwer AP, 1994.

Edmund Husserl: Briefwechsel. Familienbriefe. Hrsg. von Elisabeth Schuhmann - Karl Schuhmann. Dordrecht: Kluwer AP, 1994.

Edmund Husserl: Logische Untersuchungen. Zweiter Band. Erster Teil. Hrsg. von Ursula Panzer. The Hague: Martinus Nijhoff, 1984.

Edmund Husserl: Logische Untersuchungen. Zweiter Band. Zweiter Teil. Hrsg. von Ursula Panzer. The Hague: Martinus Nijhoff, 1984.

Edmund Husserl: Logische Untersuchungen. Ergänzungsband. Erster Teil. Hrsg. von Ullrich Melle. Dordrecht: Kluwer, 2002.

Edmund Husserl: Aufsätze und Rezensionen (1890-1910). Hrsg. von Bernhard Rang. The Hague: Martinus Nijhoff, 1979.

Edmund Husserl: Logik und allgemeine Wissenschaftstheorie. Hrsg. von Ursula Panzer. Dordrecht: Kluwer, 1996.

Edmund Husserl: Untersuchungen zur Urteilstheorie. Hrsg. von Robin D. Rollinger. Dordrecht: Springer, 2009.

Husserls Abhandlung „Intentionale Gegenstände“. Edition der ursprünglichen Druckfassung. Hrsg. von Karl Schuhmann. In: Brentano Studien 3 (1990/91), 137-176.

\section{Weitere Primärliteratur}

Anonym (1876): Programm des deutschen Staats-Obergymnasiums in Olmütz, veröffentlicht am Schlusse des Schuljahres [1875/]1876. Olmütz: Verlag der Direction des deutschen Staats- 
Author's accepted manuscript deposited in the Open Access repository (http://real.mtak.hu/) of the Hungarian Academy of Sciences (HAS) by the author under obligation imposed by the HAS, in accordance with the Copyright Transfer Agreement. Copyright 2015, All Rights Reserved by Springer Science+Business Media Dordrecht. Publishing on other websites is not covered by the exemption granted to the author by the Copyright Transfer Agreement. Scientific use only. Warning: There are discrepancies between this version and the final published one.

Published in Husserl Studies 31/2 (2015), pp. 95-121.

"The final publication is available at Springer via http://dx.doi.org/10.1007/s10743-014-9155-z"

Obergymnasiums.

Bolzano, Bernard (1810): Beyträge zu einer begründeteren Darstellung der Mathematik. Prag: Caspar Widtmann (in Husserls Bibliothek in der Neuausgabe von 1926; Signatur: BA 173).

Bolzano, Bernard (1837): Wissenschaftslehre. Versuch einer ausführlichen und größtentheils neuen Darstellung der Logik mit steter Rücksicht auf deren bisherige Bearbeiter. Sulzbach: Seidel (in Husserls Bibliothek; Signatur: BQ 46/1-4).

Brentano, Franz (1874): Psychologie vom empirischen Standpunkte. Erster Band. Leipzig: Duncker \& Humblot (in Husserls Bibliothek; Signatur: BQ 60).

Brentano, Franz (1929): Über die Zukunft der Philosophie. Hrsg. von Oskar Kraus. Leipzig: Meiner.

Brentano, Franz (1930): Wahrheit und Evidenz. Hrsg. von Oskar Kraus. Leipzig: Meiner (in Husserls Bibliothek; Signatur: BQ 69).

Brentano, Franz (1977): Die Abkehr vom Nichtrealen. Hrsg. von Franziska Mayer-Hillebrand. Hamburg: Meiner.

Brentano, Franz (1989): Briefe an Carl Stumpf. 1867-1917. Graz: Akademische Druck- u. Verlagsanstalt.

Brück, Maria (1933): Über das Verhältnis Edmund Husserls zu Franz Brentano. Vornehmlich mit Rücksicht auf Brentanos Psychologie. Inaugural-Dissertation (Rheinische Friedrich-WilhelmsUniversität Bonn). Würzburg: Konrad Triltsch.

Herbart, Johann Friedrich (1813): Lehrbuch zur Einleitung in die Philosophie. Königsberg: August Wilhelm Unzer.

Herbart, Johann Friedrich (1850): Schriften zur Einleitung in die Psychologie. Hrsg. von Gustav Hartenstein. Leipzig: Voss (in Husserls Bibliothek in der Ausgabe von 1883; Signatur: BQ 180).

Höfler, Alois (1890): Philosophische Propädeutik. I. Theil: Logik. Unter Mitwirkung von Alexius Meinong. Prag/Wien: F. Tempsky, Leipzig: G. Freytag, Leipzig (in Husserls Bibliothek, Signatur: BQ 193).

Höfler, Alois (1921): Die Philosophie des Alois Höfler. In: Raymund Schmidt (Hrsg.): Die deutsche Philosophie der Gegenwart in Selbstdarstellungen. 2. Band. Leipzig: Meiner, 117-160. 
Author's accepted manuscript deposited in the Open Access repository (http://real.mtak.hu/) of the Hungarian Academy of Sciences (HAS) by the author under obligation imposed by the HAS, in accordance with the Copyright Transfer Agreement. Copyright 2015, All Rights Reserved by Springer Science+Business Media Dordrecht. Publishing on other websites is not covered by the exemption granted to the author by the Copyright Transfer Agreement. Scientific use only. Warning: There are discrepancies between this version and the final published one.

Published in Husserl Studies 31/2 (2015), pp. 95-121.

"The final publication is available at Springer via http://dx.doi.org/10.1007/s10743-014-9155-z"

Kern, Franz (1888): Die Deutsche Satzlehre. Eine Untersuchung ihrer Grundlagen. Zweite vermehrte Auflage. Berlin: Nicolai.

Kraus, Oskar (1919): Franz Brentano. Zur Kenntnis seines Lebens und seiner Lehre. Mit Beiträgen von Carl Stumpf und Edmund Husserl. München: Oskar Beck.

Kraus, Oskar (1935): Besonderheit und Aufgabe der deutschen Philosophie in Böhmen. In: Oskar Engländer et al. (Hrsg.): Zur Philosophie der Gegenwart. Prag: Calve, 51-56.

Lindner, Gustav Adolf (1872): Lehrbuch der formalen Logik. Dritte erweiterte Auflage. Wien: Carl Gerold's Sohn (siehe Anm. 8. über Husserls Besitz dieses Buchs).

Lotze, Hermann (2003): Briefe und Dokumente. Hrsg. von Ernst Wolfgang Orth - Reinhard Pester. Würzburg: Königshausen \& Neumann.

Marty, Anton (2011): Deskriptive Psychologie. Hrsg. von Mauro Antonelli - Johann Christian Marek. Würzburg: Königshausen \& Neumann.

Osborn, Andrew Delbridge (1934): The Philosophy of Edmund Husserl in Its Development From His Mathematical Interests to His First Conception of Phenomenology in Logical Investigations. Dissertation (Columbia University, New York City) (in Husserls Bibliothek, Signatur: BP 190).

Pokorny, Alois (Hrsg.) (1869): Fünfter Jahresbericht des Leopoldtstädter Communal-Real- und Obergymnasiums. Wien: Verlag des Leopoldtstädter Real- und Obergymnasiums.

Sigwart, Christoph (1889): Logik. Erster Band. Die Lehre vom Urtheil, vom Begriff und vom Schluss. Zweite durchgesehene und erweiterte Auflage. Freiburg: J. C. B. Mohr (Paul Siebeck) (in Husserls Bibliothek; Signatur: BQ 439/1).

Twardowski, Kasimir (1894): Zur Lehre vom Inhalt und Gegenstand der Vorstellungen. Eine psychologische Untersuchung. Wien: Alfred Hölder (in Husserls Bibliothek; Signatur: BQ 482).

Ueberweg, Friedrich (1882): System der Logik und Geschichte der logischen Lehren. Fünfte, verbesserte [...] Auflage. Hrsg. von Jürgen Bona Meyer. Bonn: Adolph Marcus (in Husserls Bibliothek; Signatur BQ 485).

Zimmermann, Robert (1850): Über die jetzige Stellung der Philosophie auf der Universität. [...] Olmütz: Eduard Hölzel.

Zimmermann, Robert (1852): Was erwarten wir von der Philosophie? [...] Prag: Credner \& 
Author's accepted manuscript deposited in the Open Access repository (http://real.mtak.hu/) of the Hungarian Academy of Sciences (HAS) by the author under obligation imposed by the HAS, in accordance with the Copyright Transfer Agreement. Copyright 2015, All Rights Reserved by Springer Science+Business Media Dordrecht. Publishing on other websites is not covered by the exemption granted to the author by the Copyright Transfer Agreement. Scientific use only. Warning: There are discrepancies between this version and the final published one.

Published in Husserl Studies 31/2 (2015), pp. 95-121.

"The final publication is available at Springer via http://dx.doi.org/10.1007/s10743-014-9155-z"

Kleinbub.

Zimmermann, Robert (1860): Philosophische Propädeutik. Prolegomena - Logik - Empirische Psychologie. Zur Einleitung in die Philosophie. Zweite, umgearbeitete und sehr vermehrte Auflage. Wien: Wilhelm Braumüller (in Husserls Bibliothek, Signatur: BQ 522).

Zimmermann, Robert (1861): Philosophie und Erfahrung. Eine Antrittsrede. Wien: Wilhelm Braumüller.

Zimmermann, Robert (1867): Philosophische Propädeutik. Prolegomena - Logik - Empirische Psychologie. Zur Einleitung in die Philosophie. Dritte Auflage. Wien: Wilhelm Braumüller (in Husserls Bibliothek; Signatur: BA 1932).

\section{Forschungsliteratur}

Antonelli, Mauro (2001): Seiendes, Bewußtsein, Intentionalität im Frühwerk von Franz Brentano. Freiburg: Alber.

Fabian, Reinhard (1986): Leben und Wirken von Christian v. Ehrenfels. Ein Beitrag zur intellektuellen Biographie. In: Ders. (Hrsg.): Christian von Ehrenfels. Leben und Werk. Amsterdam: Rodopi, 1-64.

Gerlach, Hans Martin - Sepp, Hans Rainer (Hrsg.): Husserl in Halle. Spurensuche im Anfang der Phänomenologie. Frankfurt: Peter Lang.

Grimm, Gerald (2009): Gustav Adolf Lindner als Wegbereiter der Pädagogik des Herbartianismus in der Habsburgermonarchie. In: Adam, E. - Grimm, G. (Hrsg.): Die Pädagogik des Herbartianismus in der Österreichisch-Ungarischen Monarchie. Wien: Lit, 21-35.

Hemecker, Wilhelm W. (1991): Vor Freud. Philosophiegeschichtliche Voraussetzungen der Psychoanalyse. München: Philosophia.

Ierna, Carlo (2006): The Beginnings of Husserl's Philosophy. Part 2: Philosophical and Mathematical Background. In: The New Yearbook for Phenomenology and Phenomenological Philosophy 6, 33-81.

Kühndel, Jan (1969): Edmund Husserls Heimat und Herkunft. In: Archiv für Geschichte der 
Author's accepted manuscript deposited in the Open Access repository (http://real.mtak.hu/) of the Hungarian Academy of Sciences (HAS) by the author under obligation imposed by the HAS, in accordance with the Copyright Transfer Agreement. Copyright 2015, All Rights Reserved by Springer Science+Business Media Dordrecht. Publishing on other websites is not covered by the exemption granted to the author by the Copyright Transfer Agreement. Scientific use only. Warning: There are discrepancies between this version and the final published one.

Published in Husserl Studies 31/2 (2015), pp. 95-121.

"The final publication is available at Springer via http://dx.doi.org/10.1007/s10743-014-9155-z"

Philosophie 51/3, 286-290.

Künne, Wolfgang (2008): Versuche über Bolzano. Sankt Augustin: Academia.

Künne, Wolfgang (2011): „Denken ist immer Etwas Denken. “ Bolzano und (der frühe) Husserl über Intentionalität. In: Konrad Cramer - Christian Beyer (Hrsg.): Edmund Husserl 1859-2009.

Berlin: De Gruyter, 77-99.

Morscher, Edgar (1997): Robert Zimmermann - der Vermittler von Bolzanos Gedankengut?

Zerstörung einer Legende. In: Heinrich Ganthaler - Otto Neumaier (Hrsg.): Bolzano und die österreichische Geistesgeschichte. Sankt Augustin: Academia, 145-236.

Rollinger, Robin D. (1999): Husserl's Position in the School of Brentano. Dordrecht: Kluwer AP.

Schuhmann, Karl (1977): Husserl-Chronik. Denk- und Lebensweg Edmund Husserls. Husserliana Dokumente 1. Den Haag: Martinus Nijhoff.

Schuhmann, Karl (Hrsg.) (1988a): Malvine Husserls „, Skizze eines Lebensbildes von E. Husserl“. In: Husserl Studies 5/2, 105-125.

Schuhmann, Karl (1988b): Husserl and Masaryk. In: Josef Novák (Hrsg.): On Masaryk. Texts in English and German. Amsterdam: Rodopi, 129-156.

Schuhmann, Karl (2004): Selected Papers in Phenomenology. Hrsg. von Cees Leijenhorst - Piet Steenbakkers. Dordrecht: Kluwer AP.

Wieser, Alfred Rhaeticus (1950): Die Geschichte des Faches Philosophie an der Universität Wien (1848-1938). Dissertation (Universität Wien).

Winter, Eduard (1975): Robert Zimmermanns Philosophische Propädeutik und die Vorlagen aus der Wissenschaftslehre Bernard Bolzanos. Wien: Verlag der Österreichischen Akademie der Wissenschaften.

Winter, Eduard (1993): Ausgewählte Schriften aus dem Nachlaß. Hrsg. von Edgar Morscher. Sankt Augustin: Academia.

Zahavi, Dan (2002): Metaphysical Neutrality in Logical Investigations. In: Dan Zahavi - Frederik Stjernfelt (Hrsg.): One Hundred Years of Phenomenology. Husserl's Logical Investigations Revisited. Dordrecht: Kluwer AP, 93-123. 